\title{
Croissance des boules et des géodésiques fermées dans les nilvariétés
}

\author{
PIERRE PANSU \\ Centre de Mathematiques, Ecole Polytechnique, 91128 Palaiseau Cedex, France
}

(Received 27 May 1982)

Abstract. If $(M, g)$ is a riemannian nilmanifold, the homothetic metrics $\varepsilon \tilde{g}$ on the universal cover $M$ converge in the sense of Gromov for small $\varepsilon$. In this convergence the volume of balls and the number of closed geodesics go to a limit, and precise asymptotic estimates are given for these numbers.

\section{A. Introduction}

Cet article porte sur la croissance des groupes discrets nilpotents, et des variétés riemanniennes sur lesquelles ils agissent par isométries.

(1). La croissance 'algébrique' d'un groupe discret, de type fini $\Gamma$, a été définie par A. Švarč [16]. Fixons un système générateur symétrique, fini $S$; tout élément $\gamma \mathrm{du}$ groupe peut s'écrire comme un mot

$$
\gamma=\prod \gamma_{i}^{p_{i}}
$$

en les générateurs $\gamma_{i} \in S$, dont la longueur est $\sum\left|p_{i}\right|$ par définition. La norme algébrique $|\gamma|$ relative au système $S$ est la longueur minimale d'un mot en les générateurs qui représente $\gamma$. Pour un réel positif $R$, notons $N_{S}(R)$ le nombre d'éléments de $\Gamma$ dont la norme est inférieure à $R$. Le type de croissance de $\Gamma$ est le type de croissance (exponentiel, polynomial) de la fonction $N_{S}(R)$.

(2) La croissance 'géométrique' d'une variété riemannienne $(\tilde{M}, \tilde{g})$ se mesure au volume des boules: nous notons $\operatorname{vol}_{\tilde{m}}(R)$ le volume de la boule de centre $\tilde{m}$, de rayon $R$.

(3) La remarque fondamentale de V. Efremovič $[2]$ est que, si $(\tilde{M}, \tilde{g})$ est le revêtement universel riemannien d'une variété compacte $(M, g)$, la croissance géométrique de $(\tilde{M}, \tilde{g})$ ne dépend que du groupe fondamental $\Gamma=\pi_{1}(M)$.

Précisément, pour tout système générateur $S$ de $\Gamma$, tout point $\tilde{m}$ de $\tilde{M}$, il existe des constantes $a, b, c$ telles que

$$
a N_{S}\left(\frac{R}{c}\right) \leq \operatorname{vol}_{\tilde{m}}(R) \leq b N_{S}(c R) .
$$

(4) Si le groupe $\Gamma$ est nilpotent, il résulte d'un théorème de J. Wolf [18] que cette croissance est polynomiale.

Plus précisément, d'après $H$. Bass [1], il existe des constantes $c$ et $C$ (dépendant du système générateur fini $S$ ) telles que

$$
c R^{p} \leq N_{S}(R) \leq C(R+1)^{p}
$$


où l'exposant $p$ est un entier, que nous appelerons dimension homogène du groupe discret $\Gamma$, défini comme suit: notons

$$
\Gamma^{1}=\Gamma=\pi_{1}(M), \quad \Gamma^{2}=[\Gamma, \Gamma], \quad \Gamma^{i}=\left[\Gamma, \Gamma^{i-1}\right]
$$

les sous-groupes de commutateurs successifs; les quotients $\Gamma^{i} / \Gamma^{i+1}$ sont abéliens, de type fini, et l'on a

$$
p=\sum_{i=1}^{\infty} i \operatorname{rang}\left(\Gamma^{i} / \Gamma^{i+1}\right) .
$$

(5) Nous nous proposons de raffiner encore le résultat de H. Bass.

Proposition (\$51). Si le groupe discret, de type fini, $\Gamma$ est presque nilpotent (i.e. admet un sous-groupe nilpotent d'indice fini), la limite

$$
\lim _{R \rightarrow \infty} N_{S}(R) R^{-p}
$$

existe toujours. Si le groupe fondamental de la variété compacte $(M, g)$ est presque nilpotent, la limite

$$
\lim _{R \rightarrow \infty} \operatorname{vol}_{\bar{m}}(R) R^{-p}
$$

existe toujours, elle ne dépend pas du point $\tilde{m}$ dans le révêtement universel $(\tilde{M}, \tilde{g})$, seulement de la métrique $g$.

(6) L'intérêt de ce papier réside plus dans la méthode employée que dans le résultat lui-même. Alors que la démarche de Wolf et Bass (voir aussi [17]) a une saveur combinatoire, nous décrivons, au moyen d'un 'espace limite', le comportement géométrique à l'infini du revêtement universel riemannien $(\tilde{M}, \tilde{g})$.

Il s'agit de mettre en évidence un comportement analogue à celui de l'espace euclidien $\left(\mathbb{R}^{n}\right.$, can): celui-ci possède des homothéties; ce sont des homéomorphismes $\delta_{t}: \mathbb{R}^{n} \rightarrow \mathbb{R}^{n}$ tels que

$$
\delta_{t}^{*} \operatorname{can}=t^{2} \text { can. }
$$

Autrement dit, $\delta_{t}$ réalise une isométrie de $\left(\mathbb{R}^{n}\right.$, can) sur $\left(\mathbb{R}^{n}, t^{2}\right.$ can $)$.

Én général, $(\tilde{M}, \tilde{g})$ n'est pas isométrique à $\left(\tilde{M}, t^{2} \tilde{g}\right)$, pour $t \neq 1$, mais n'est pas loin de lui être isométrique: nous montrons que, lorsque $\varepsilon$ tend vers 0 , les espaces $\left(\tilde{M}, \varepsilon^{2} \tilde{g}\right)$ convergent, en un sens dû à Gromov, vers un espace limite $\left(G_{\infty}, d_{\infty}\right)$, qui, lui, admet des homothéties.

(7) Définition. ([7] ou $[8,3.4])$ Soient $X$ et $Y$ deux espaces métriques compacts. La distance de Hausdorff

$$
\operatorname{dist}_{H}(X, Y)
$$

est la borne inférieure des $\varepsilon>0$ tels qu'il existe des plongements isométriques de $X$ et $Y$ dans un espace métrique $Z$, avec la propriété suivante: $X$ est dans le $\varepsilon$-voisinage de $Y$, et $Y$ est dans le $\varepsilon$-voisinage de $X$.

dist $_{H}$ est une distance sur les classes d'espaces métriques isométriques.

(8) Concrètement, pour montrer que $\operatorname{dist}_{H}(X, Y)$ est petit, il suffit $([\mathbf{8}, 3.5])$ d'exhiber des parties finies $\left\{x_{1}, \ldots, x_{N}\right\} \subset X$, et $\left\{y_{1}, \ldots, y_{N}\right\} \subset Y$, telles que: 
la suite $x_{i}$ est assez dense dans $X$, i.e. $X$ est un $\varepsilon$-voisinage de $\left\{x_{i}\right\}$ pour un petit $\varepsilon$;

la suite $y_{i}$ est assez dense dans $Y$;

tous les rapports $d\left(y_{i}, y_{j}\right) / d\left(x_{i}, x_{j}\right)$ sont proches de 1 .

Nous faisons référence à $[\mathbf{8}]$ pour les propriétés et exemples de convergence de Hausdorff.

(9) Nous avons affaire à des espaces non compacts, mais à boules compactes. Nous utilisons la notion de 'convergence au sens de Hausdorff pointé'.

Définition. ([8, 3.14]) Pointons les $X_{i}$ en $x_{i} \in X_{i}$ et $X$ en $x \in X$. On dit que les espaces métriques $X_{i}$ convergent au sens de Hausdorff pointé vers $X$ si, pour tout $R$, il existe une suite $\varepsilon_{i}$ tendent vers 0 telle que les boules

$$
B\left(x_{i}, R+\varepsilon_{i}\right) \subset X_{i}
$$

convergent, au sens de la distance de Hausdorff, vers la boule $B(x, R)$, de centre $x$ et de rayon $R$ dans $X$.

Remarque. Cette notion entraîne la convergence au sens de la 'distance de Hausdorff modifiée' de [7].

(10) ThÉORÈME PRINCIPAL. Soit $M$ une variété compacte, dont le groupe fondamental $\Gamma$ est presque nilpotent. Munissons $\Gamma$ d'une norme | | (i.e. d'une distance invariante à gauche), induite:

ou bien, par le choix d'un système générateur symétrique $S$;

ou bien, par le choix d'une métrique riemannienne $g$ sur $M$ et d'un point $\tilde{m}$ de $\tilde{M}$, c'est-à-dire,

$$
|\gamma|=d_{\tilde{g}}(\tilde{m}, \gamma \tilde{m})
$$

Lorsque $\varepsilon$ tend vers 0 , les espaces métriques homothétiques $(\Gamma, \varepsilon||)$ convergent, au sens de Hausdorff pointé, vers un espace métrique $\left(G_{\infty}, d_{\infty}\right)$, où $G_{\infty}$ est un groupe de Lie nilpotent, et $d_{\infty}$ est une distance invariante à gauche sur $G_{\infty}$.

Ce théorème est démontré au $\$ 49$.

Remarque. Comme l'orbite $\Gamma \tilde{m}$ est de plus en plus dense dans $\left(\tilde{M}, \varepsilon^{2} \tilde{g}\right)$, lorsque $\varepsilon$ tend vers 0 , le théorème principal entraîne la convergence des espaces $\left(\tilde{M}, \varepsilon^{2} \tilde{g}\right)$ vers $\left(G_{\infty}, d_{\infty}\right)$.

(11) Le théorème principal est simplement l'extension aux groupes presque nílpotents généraux des résultats de:

$[7, \S 7]$, pour la norme algébrique associée à un système de deux générateurs et leurs opposés, sur $\Gamma=\mathbb{Z}^{2}$;

$[7, \S 7]$, pour une métrique riemannienne $g$, invariante à gauche sur le groupe de Lie nilpotent de dimension 3 ;

$[8,3.16]$, pour une variété riemannienne dont le groupe fondamental est abélien libre.

On peut le considérer comme une illustration de l'article [7] de M. Gromov sur les groupes à croissance polynomiale. Pour montrer qu'un tel groupe est presque nilpotent, M. Gromov prouve que, pour toute norme algébrique | |, il existe des 
sous-suites $\left(\Gamma, \varepsilon_{i}||\right), \varepsilon_{i}$ tendant vers 0 , qui convergent au sens de Hausdorff. Notre théorème apporte une description de l'espace limite commun à toutes ces suites.

(12) Remarque. Revenons au problème de croissance des groupes discrets. Le $\S 5$ suggère une question générale. Soit $\Gamma$ le groupe fondamental de la variété compacte $M$, agissant par isométries sur le revêtement universel riemannien $(\tilde{M}, \tilde{g})$. Notons $N_{g}(R)$ le nombre de points d'une orbite se trouvant dans une boule de rayon $R$.

'Existe-t-il une fonction $f_{\Gamma}$ telle que $f_{\Gamma}(N g(R), R)$ converge pour toute métrique riemannienne $g$ ?'

G. A. Margulis a analysé le cas des métriques à courbure négative dans [13]. Ses résultats suggèrent une réponse positive à la question ci-dessus pour les groupes fondamentaux des variétés compactes qui admettent une métrique à courbure négative. G. A. Margulis était motivé par les classiques 'problèmes de réseaux' de la' théorie algébrique des nombres, c.f. [19].

L'énoncé du théorème principal de ce papier a été proposé par A. Douady, à l'occasion d'une série d'exposés sur l'article 'Groups of Polynomial Growth and Expanding Maps'. A. Douady m'a aidé à le démontrer; en particulier, il m'a appris l'élégante construction du $\S 39$, où un groupe nilpotent apparaît comme une déformation de son gradué. Qu'il en soit ici très vivement remercié.

Je tiens aussi à remercier M. Gromov, qui a dégagé les conséquences géométriques de la convergence des groupes homothétiques.

\section{B. Description de l'espace limite}

(13) De cet espace métrique $Y$, nous savons, par les théorèmes généraux:

que la distance de deux points est égale à longueur minimum d'un chemin qui les relie;

qu'il admet un groupe à un paramètre d'homothéties $\delta_{t}$;

que son groupe d'isométries est un groupe de Lie transitif sur $Y$.

Ceci rend plausible le fait que $Y$ soit lui-même un groupe de Lie $G_{\infty}$. L'homogénéïté 'géométrique' (existence d'homothéties) de l'espace $Y$ se traduit par une 'homogénéité algébrique' du groupe $G_{\infty}$ : les homothéties $\delta_{t}$ sont des automorphismes du groupe $G_{00}$, qui admettent une base de vecteurs propres dans l'algébre de Lie. Dans une telle base, la matrice de $\delta_{t}$ est

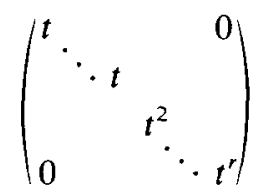

L'existence de tels automorphismes équivaut à la propriété suivante:

(14) Définition. ([6]) Le groupe de Lie $G$ est dit gradué si son algèbre de Lie admet une graduation, c'est-à-dire, une décomposition

$$
\mathscr{G}=V_{1} \oplus \cdots \oplus V_{r}
$$

telle que

(i) $V_{i}$ est un supplémentaire de $\mathscr{G}^{i+1}$ dans $\mathscr{G}^{i}$; 
(ii) $\left[V_{i}, V_{j}\right] \subset V_{i+j}$;

où on a posé

$$
\mathscr{G}^{1}=\mathscr{G}, \quad \mathscr{G}^{i}=\left[\mathscr{G}, \mathscr{G}^{i-1}\right] .
$$

Par exemple, toute algèbre de Lie nilpotente d'ordre 2 admet les graduations $V \oplus \mathscr{G}^{2}$, où $V$ est un supplémentaire quelconque de $\mathscr{G}^{2}$ dans $\mathscr{G}$. Il existe des groupes nilpotents non gradués dès la dimension 6 (voir [6]).

A toute graduation $V_{i}$ est attaché un groupe à un paramètre d'automorphismes

$$
\delta_{t}: \delta_{t}(x)=t^{i} x \quad \text { si } x \in V_{i} \text {. }
$$

Inversement, si le groupe d'automorphismes $\delta_{t}$ est diagonalisable, les espaces propres forment une graduation.

(15) En fait, le groupe de Lie limite $G_{\infty}$ ne dépend que de la structure du groupe $\Gamma$. Il est construit comme suit: par hypothèse, il existe dans $\Gamma$ un sous-groupe nilpotent $N$ d'indice fini; notons

$$
\Gamma^{\prime}=N / \text { tor } N
$$

alors $\Gamma^{\prime}$ est sans torsion; d'après Malcev [12], $\Gamma^{\prime}$ est isomorphe à un sous-groupe discret cocompact d'un groupe de Lie nilpotent $G$; alors $G_{\infty}$ est le groupe gradué associé à $G$, i.e. le groupe de Lie simplement connexe dont l'algèbre de Lie est l'algèbre graduée $\mathscr{G}_{\infty}$ associée à $\mathscr{G}$.

(16) Définition. L'algèbre graduée associée à l'algèbre de Lie $\mathscr{G}$ est la somme directe

$$
\mathscr{G}_{\infty}=\bigoplus_{i=1}^{r} \mathscr{G}^{i} / \mathscr{G}^{i+1},
$$

munie du crochet suivant: si $x \in \mathscr{G}^{i}, y \in \mathscr{G}^{j}$, le crochet

$$
[\bar{x}, \bar{y}]=\overline{[x, y]}
$$

est bien défini modulo $\mathscr{G}^{i+j+1}$.

(17) Description de la métrique $d_{\infty}$. L'existence des homothéties $\delta_{t}$ donne une idée de la forme des boules dans l'espace limite $Y$ : les boules de faible rayon sont plus aplaties que les boules de même rayon relatives à une métrique riemannienne sur $G_{\infty}=Y$.

De façon précise, notons

$$
\log : G_{\infty} \rightarrow \mathscr{G}_{\infty}
$$

le difféomorphisme réciproque de l'exponentielle; notons $\pi^{\prime}$ la projection sur les facteurs $V_{2} \oplus \cdots \oplus V_{r}$ de la graduation associée aux $\delta_{i}$; notons $\nu$ une norme sur ces facteurs.

(18) LeMme. Si $x \in G_{\infty}$ et si $d_{\infty}(1, x) \leq 1$, alors

$$
\nu\left(\pi^{\prime} \log x\right) \leq \text { const. } d_{\infty}(1, x)^{2} .
$$

Démonstration. En effet, pour un $z$ dans la boule unité $B_{\infty}(1)$, écrivons

$$
\log z=z_{1}+\cdots+z_{r}
$$

d'où

$$
\log \delta_{\varepsilon}(z)=\delta_{\varepsilon} \log z=\varepsilon z_{1}+\cdots+\varepsilon^{r} z_{r}
$$


et

$$
\pi^{\prime} \delta_{\varepsilon}(\log z) / \varepsilon^{2}=z_{2}+\cdots+\varepsilon^{r-2} z_{r}
$$

est borné pour $\varepsilon \leqslant 1$ et $z \in B_{\infty}(1)$. Il vient donc

$$
\nu\left(\pi^{\prime} \log x\right) \leq \text { const. } \varepsilon^{2} \quad \text { pour } x \in B_{\infty}(\varepsilon) .
$$

Il résulte de ce lemme que, si une courbe $c(s)$ absolument continue a une longueur

$$
\text { longueur }(c)=\sup \sum d_{\infty}\left(c\left(s_{i}\right), c\left(s_{i+1}\right)\right)
$$

finie, alors $c(s)$ est presque partout tangente au champ de plans obtenu en translatant le sous-espace $V_{1}$ à gauche. On peut donc s'attendre à ce que la distance $d_{\infty}$ soit une métrique de Carnot-Carathéodory.

(19) Définition. Soit $M$ une variété, soit $V$ un sous-fibré du fibré tangent à $M$, muni d'une norme sur les fibres. Pour une courbe absolument continue $c(s)$, presque partout tangente à $V$, on pose

$$
\text { longueur }(c)=\int\left\|\frac{d c}{d s}\right\| d s .
$$

La métrique de Carnot-Carathéodory associée au sous-fibré normé $V$ est donnée par $d(x, y)=\inf \{$ longueur $(c): c$ relie $x$ à $y$, absolument continue, tangente à $V\}$.

(20) La théorie du contrôle ([15]) donne des conditions suffisantes pour qu'une telle distance soit finie. Ici, le sous-fibré est obtenu en translatant à gauche l'espace $V_{1}$, et ces conditions sont remplies: les champs de vecteurs tangents à $V_{1}$ (en particulier, les champs invariants à gauche) et leurs crochets successifs engendrent l'espace tangent à $G_{\infty}$ en tout point. Plus précisément,

THÉorÈmE. ([15]) La topologie induite par $d_{\infty}$ sur $G_{\infty}$ est sa topologie ordinaire de variété.

Pour plus d'information sur les métriques de Carnot sur les groupes nilpotents, voir [4], [5], [10], [11], [14].

(21) Construction de la norme sur le sous-fibré $V_{1}$. La norme initiale | | sur $\Gamma$ induit une norme quotient sur le sous-groupe abélien

$$
A=\Gamma^{\prime} /\left[\Gamma^{\prime}, \Gamma^{\prime}\right]
$$

de l'espace vectoriel $B=G /[G, G]$. On rend cette norme homogène en posant

$$
|a|_{\infty}=\lim _{k \rightarrow \infty} \frac{1}{k}|k a| \text {. }
$$

La fonction | $\left.\right|_{\infty}$ se prolonge à $B$ et en fait un espace de Banach. Pour toute graduation $V_{1} \oplus \cdots \oplus V_{r}$ de $\mathscr{G}_{\infty}$, la projection

$$
\pi: G_{\infty} \rightarrow G_{\infty} /\left[G_{\infty}, G_{\infty}\right]
$$

induit un isomorphisme de $V_{1}$ sur $G /[G, G]=B$, grâce auquel on transporte la norme ||$_{\infty}$ sur $V_{1}$.

(22) Remarque. L'espace métrique obtenu $\left(G_{\infty}, d_{\infty}\right)$ est indépendant du choix de la graduation $V_{i}$ dans $\mathscr{G}_{\infty}$. En effet, si $V_{i}^{\prime}$ est une autre graduation, l'unique 
application linéaire $\phi: \mathscr{G}_{\infty} \rightarrow \mathscr{G}_{\infty}$ telle que

(i) $\phi\left(V_{i}\right)=V_{i}^{\prime}$;

(ii) $\phi$ induit l'identité sur $\mathscr{G}_{\infty}^{i} / \mathscr{G}_{\infty}^{i+1}$;

est un automorphisme de $\mathscr{G}_{\infty}$, donc fournit une isométrie de $\left(G_{\infty}, d_{\infty}\right) \operatorname{sur}\left(G_{\infty}, d_{\infty}^{\prime}\right)$.

(23) Remarque. Cas d'une norme algébrique associée à un système générateur symétrique $S$, sur un sous-groupe $\Gamma$ discret et cocompact dans $G$ : la boule unité de | $\left.\right|_{\infty}$ dans $B=G /[G, G]$ est l'enveloppe convexe de $\pi(S)$.

(24) Remarque. Cas d'une norme associée à une métrique riemannienne sur $M$. L'homomorphisme d'Hurewicz induit un plongement de $\Gamma /[\Gamma, \Gamma]$ comme sousgroupe discret cocompact de $H_{1}(M, \mathbb{R})$, donc transporte la 'norme limite' | $\left.\right|_{\infty}$ sur $H_{1}(M, \mathbb{R})$.

Or Federer et Fleming [3] définissent une 'norme stable' sur $H_{1}(M, \mathbb{R})$ : d'abord, pour une 1-chaîne

$$
c=\sum \lambda_{i} \sigma_{i}
$$

à coefficients réels, où les $\sigma_{i}$ sont des chemins lipschitziens dans $M$, ils définissent la masse

$$
M(c)=\sum\left|\lambda_{i}\right| \text { longueur }\left(\sigma_{i}\right)
$$

la norme stable $\left|\alpha_{R}\right|$ d'une classe d'homologie réelle $\alpha$ est la borne inférieure des masses $M(c)$ des cycles $c$ représentant $\alpha$. Il résulte de $\left[8,4.20\right.$ bis], que notre | $\left.\right|_{\infty}$ coïncide avec cette norme stable.

(25) Nous pouvons donc ajouter un

Complément au théorème principal. Le groupe de Lie $G_{\infty}$ est gradué. La distance $d_{\infty}$ est une métrique de Carnot-Carathéodory associée à un sous-fibré invariant à gauche $V_{1}$; celui-ci est obtenu en translatant le premier sous-espace (supplémentaire des commutateurs $\left.\left[\mathscr{G}_{\infty}, \mathscr{G}_{\infty}\right]\right)$ d'une graduation de l'algèbre de Lie $\mathscr{G}_{\infty}$. Ces objets ne dépendent que de la structure du groupe $\Gamma$; en particulier,

$$
\begin{aligned}
\operatorname{dim} G_{\infty} & =\sum_{i} \operatorname{rang}\left(\Gamma^{i} / \Gamma^{i+1}\right) \\
\operatorname{dim} V_{1} & =\operatorname{rang}(\Gamma /[\Gamma, \Gamma]) .
\end{aligned}
$$

Seule la norme sur $V_{1}$ dépend du système générateur $S$ (de la métrique riemannienne g, respectivement).

\section{Schéma de démonstration}

(26) Plaçons nous dans le cas simple où le groupe $\Gamma$ est discret cocompact dans un groupe de Lie gradué $G$. A une norme | | sur $\Gamma$, algébrique ou géométrique, nous avons associé au $\S 21$ une distance de Carnot-Carathéodory $d_{\infty}$ sur $G_{\infty}=G$. Celle-ci réalise, asymptotiquement, une bonne approximation de la norme donnée sur $\Gamma$ :

Proposition 41. Pour $\varepsilon$ tendant vers 0 , soit $\gamma_{\varepsilon}$ un élément de $\Gamma$ tel que $\delta_{\varepsilon} \gamma_{\varepsilon}$ tende vers $x \in G, x \neq 1$. Alors $\varepsilon\left|\gamma_{\varepsilon}\right|$ tend vers $d_{\infty}(1, x)$.

(27) Le théorème principal en découle. En effet, notons

$$
\Gamma^{r}=\{\gamma \in \Gamma:|\gamma| \leq r\}
$$


la boule de rayon $r$ dans $\Gamma$. Il s'agit de montrer que, lorsque $\varepsilon$ tend vers 0 , la boule $\Gamma^{R / \varepsilon}$, munie de la métrique $\varepsilon||$, converge au sens de Hausdorff vers la boule $B_{\infty}(R)$ de rayon $R$ dans $\left(G, d_{\infty}\right)$.

Nous utilisons le critère des réseaux ( $\$ 8)$. Fixons un $\eta$-réseau $N_{\eta}$ dans $B_{\infty}(R)$. Notons $D$ le diamètre, relatif à $d_{\infty}$, d'un domaine fondamental $P$ pour l'action de $\Gamma$ dans $G$. Pour chaque $x$ de $N_{\eta}$, notons $h_{\varepsilon}(x)$ l'élément $\gamma$ de $\Gamma$ tel que

$$
\delta_{1 / \varepsilon} x \in \gamma P
$$

Alors $h_{\varepsilon}$ est presque une isométrie de $\left(N_{\eta}, d_{\infty}\right)$ sur $\left(h_{\varepsilon}\left(N_{\eta}\right), \varepsilon||\right)$.

En effet, si $x \neq x^{\prime} \in N$, si $\gamma=h_{\varepsilon}(x), \gamma^{\prime}=h_{\varepsilon}\left(x^{\prime}\right)$, alors $\delta_{\varepsilon}\left(\gamma^{-1} \gamma^{\prime}\right)$ tend vers $x^{-1} x^{\prime}$, donc $\varepsilon\left|\gamma^{-1} \gamma^{\prime}\right| / d_{\infty}\left(x, x^{\prime}\right)$ tend vers 1 .

Pour tout $x \in N_{\eta},\left|h_{\varepsilon}(x)\right| / d_{\infty}(1, x)$ tend vers 1 , donc $h_{\varepsilon}\left(N_{\eta}\right)$, est contenu dans $\Gamma^{(R+\theta) / \varepsilon}$, où $\theta$ tend vers 0 lorsque $\varepsilon$ tend vers 0 . Inversement, si $\gamma \in \Gamma^{(R+\theta) / \varepsilon}$, alors $\delta_{\varepsilon} \gamma \in B_{\infty}\left(R+\theta^{\prime}\right)$ pour un $\theta^{\prime}$ un peu plus grand que $\theta$. Il existe donc un $x \in N_{\eta}$ tel que

$$
d_{\infty}\left(x, \delta_{\varepsilon} \gamma\right) \leq \eta+\theta^{\prime}
$$

alors

$$
d_{\infty}\left(\gamma, h_{\varepsilon}(x)\right) \leq D+\left(\eta+\theta^{\prime}\right) / \varepsilon
$$

d'où

$$
\varepsilon\left|\gamma^{-1} h_{\varepsilon}(x)\right| \leq 2 \eta
$$

pour $\varepsilon$ assez petit, ce qui prouve que $h_{\varepsilon}\left(N_{\eta}\right)$ est un $2 \eta$-réseau de $\left(\Gamma^{(R+\theta) / \varepsilon}, \varepsilon||\right)$. Voir $\$ 49$.

28. Demonstration de la proposition 41. ( $\$ 44$ à $\$ 46$ ). Les normes algébrique et géométrique partagent la propriété suivante (dite 'de longueur', voir définition 34): si $|\gamma|$ est très grand, il existe un 'géodésique discrète'

$$
\gamma_{1}, \gamma_{1} \gamma_{2}, \ldots, \gamma_{1} \gamma_{2} \ldots \gamma_{k}=\gamma
$$

dans $\Gamma$, de vitesse sup $\left|\gamma_{i}\right|$ bornée, de longueur $\sum\left|\gamma_{i}\right|$ proche de $|\gamma|$.

Si les $\delta_{\varepsilon} \gamma_{\varepsilon}$ convergent vers $x \neq 1$, les 'géodésiques discrètes' $\delta_{\varepsilon} \prod_{i} \gamma_{\varepsilon}^{i}$ (ou au moins une sous-suite) convergent uniformément vers une vraie courbe $c$ reliant 1 à $x$ dans $G$. Comme $\sup \left|\gamma_{\varepsilon}^{i}\right|$ est borné, les $\delta_{\varepsilon} \gamma_{\varepsilon}^{i}$ sont de plus en plus horizontaux (i.e. proches de $V_{1}$ ), donc la courbe $c$ est tangente à $V_{1}$. Cela prouve que

$$
\liminf _{\varepsilon \rightarrow 0} \varepsilon\left|\gamma_{\varepsilon}\right| \geq \text { longueur }_{\infty}(c) \geq d_{\infty}(1, x)
$$

Inversement, si $c$ est une géodésique minimisante de 1 à $x$, on peut approcher $\delta_{1 / \varepsilon} c$ par une 'courbe discrète' $\prod_{i} \gamma_{\varepsilon}^{i}$ dans $\Gamma$, d'où

$$
\limsup _{\varepsilon \rightarrow 0} \varepsilon\left|\gamma_{\varepsilon}\right| \leq \text { longueur }(c)=d_{\infty}(1, x)
$$

\section{Applications du théorème principal}

Ce sont plutôt des conséquences de la proposition 41, qui permet, lorsque des grandes distances sont en jeu, de remplacer la norme géométrique | | sur $\Gamma$ par $d_{\infty}$. 
(29) Volume des boules. Etant donnée une métrique riemannienne $g$ sur la variété compacte $M$, et un point $\tilde{m}$ du revêtement universel $\tilde{M}$, le rapport

$$
\operatorname{vol}_{\tilde{m}}(R) / \operatorname{vol}(g)
$$

est environ le nombre de points $\gamma \tilde{\boldsymbol{m}}$ de l'orbite, avec $|\gamma| \leq R$, d'où

$$
\frac{\operatorname{vol}_{\bar{m}}(R)}{\operatorname{vol}(g)} \sim \frac{\mu B_{\infty}(R)}{\mu(P)}=R^{p} \frac{\mu B_{\infty}(1)}{\mu(P)},
$$

où $\mu$ est une mesure de Haar sur $G_{\infty}$ ou $G$ (voir $\S 51$ ) et $P$ est un domaine fondamental pour $\Gamma^{\prime}$ dans $G$. On en déduit ( $\left.\$ 51\right)$ le théorème et la définition suivants:

Proposition. La limite

$$
\operatorname{vol}_{\infty}(g)=\lim _{R \rightarrow \infty} R^{-p} \operatorname{vol}_{\tilde{m}}(R)
$$

existe toujours; elle ne dépend pas du point $\tilde{m}$.

Nous l'appellerons volume asymptotique de $g$.

(30) Nous allons expliciter le volume asymptotique dans des cas simples. De façon à faire le lien avec l'article de M. Gromov [9], rappelons que la variété de Jacobi de $(M, g)$ est le tore

$$
J_{1} M=H_{1}(M, \mathbb{R}) / H_{1}(M, \mathbb{Z}),
$$

muni de la métrique de Finsler induite par la norme stable $\left.\right|_{\mathbb{R}} \mid$ (c.f. $\left.\$ 24\right)$. Gromov définit une normalization, qu'il note mass*, de la mesure de Lebesgue sur $J_{1} M$ $([9,4.1])$.

(31) Cas d'un tore. Il vient

$$
\operatorname{vol}_{\infty}(g)=\mu^{*} \frac{\operatorname{vol}(g)}{\text { mass* }\left(J_{1} M\right)}
$$

La constante $\mu^{*}$, volume, pour mass*, de la boule unité de $|\mathbb{R}|$, ne dépend que de cette norme. Gromov ([9,7.5.A]) sait minorer le volume asymptotique: $\operatorname{si} \operatorname{dim} M=$ $n$,

$$
\operatorname{vol}_{\infty}(g) \geq \frac{2^{n}}{n !}
$$

Il est clair, en revanche, qu'en faisant une bosse dans un tore plat, on peut rendre le volume arbitrairement grand, sans changer beaucoup la variété de Jacobi. Le volume asymptotique n'est donc pas borné.

(32) Cas d'une nilvariété de dimension 3: $M=\Gamma \backslash G$. Les sous-groupes discrets cocompacts du groupe nilpotent $G$ de dimension 3 - le groupe de Heisenberg - sont classés par un entier naturel $k$ : c'est l'indice minimum d'un sous-groupe à deux générateurs. Avec les notations de [9],

Proposition. (\$ 58). $\operatorname{vol}_{\infty}(g)=k \nu^{*} \operatorname{vol}(g)\left(\text { mass }^{*} J_{1} M\right)^{-2}$, où la constante $\nu^{*}$-volume de la boule unité relative à la métrique de CarnotCarathéodory - ne dépend que de la norme stable $\left.\right|_{\mathbb{R}} \mid$. Elle vérifie

$$
\frac{1}{4} \nu^{* 1} \leq \nu^{*} \leq \nu^{* 1}, \quad \text { où } \nu^{* 1}=\frac{2307}{2^{5} 3^{4}}=0.89 .
$$


Dans [9, 7.5.C], M. Gromov minore le volume asymptotique d'une nilvariété de dimension 3 en fonction d'autres invariants géométriques.

(33) Géodésiques périodiques. Pour $R$ assez grand, la variété riemannienne $(M, g)$ peut avoir une infinité de géodésiques périodiques de longueur inférieure à $R$. Il y en a cependant un nombre minimum, dont l'existence est forcée par la structure du groupe fondamental $\pi_{1}(M)$ : appelons 'nombre topologique de géodésiques fermées de longueur inférieure à $R$ ' le nombre $N_{c}(R)$ de classes de conjugaison dans $\pi_{1}(M)$ qui sont représentées par une courbe fermée de longueur inférieure à $R$. Dans le cas où $\pi_{1}(M)$ est abélien libre de rang $p$, la limite

$$
\lim _{R \rightarrow \infty} N_{c}(R) R^{-p}
$$

existe (elle est non nulle), cela résulte de [8, 3.16].

PROPOSITION. ( $\$ 66)$ Si $\pi_{1}(M)$ est isomorphe à un sous-groupe discret cocompact du groupe de Heisenberg, i.e. du groupe de Lie nilpotent de dimension 3, la limite

$$
\lim _{R \rightarrow \infty} N_{c}(R) / R^{2}
$$

existe, elle est positive.

\section{E. Propriétés de la norme limite | $\left.\right|_{\infty}$}

De façon à traiter simultanément le cas d'une norme 'algébrique' ou 'géométrique' sur un groupe discret $\Gamma$, nous introduisons une classe de normes-i.e. de distances invariantes à gauche-qui les contient.

(34) Définition. Une norme / | sur un groupe discret de type fini $\Gamma$ est dite 'de longueur' si

(i) $|\gamma|=\left|\gamma^{-1}\right|$;

(ii) pour tout $\varepsilon>0$, il existe un $p$ tel que tout $\gamma \in \Gamma$ puisse s'écrire comme un $\operatorname{mot} \prod \gamma_{i}$, où

$$
\left|\gamma_{i}\right| \leq p, \quad \text { et } \sum\left|\gamma_{i}\right| \leq(1+\varepsilon)|\gamma| .
$$

Clairement, la norme 'de mots' associée à un système générateur $S$ symétrique est de longueur, avec $p=1$ pour tout $\varepsilon$. La norme $|\gamma|=d_{\tilde{g}}(\tilde{m}, \gamma \tilde{m})$ induite sur $\pi_{1}(M, m)$ par la métrique riemannienne $g$ est de longueur, avec $p=2\left(1+\varepsilon^{-1}\right)$ diamètre $(g)$.

Toutes ces normes sont deux à deux équivalentes: en effet, étant donnée une norme | | de longueur, notons $S=\{\gamma:|\gamma|<p\}$, notons ||$s$ la norme de mots associée à ce système générateur (si $p$ est assez grand); alors pour tout $\gamma$ :

$$
\frac{1}{p}|\gamma| \leq|\gamma|_{s} \leq(1+\varepsilon)|\gamma| \text {. }
$$

Si $F$ est un sous-groupe distingué fini de $\Gamma$, si $N$ est un sous-groupe d'indice fini de $\Gamma$, alors une norme de longueur sur $\Gamma$ induit par restriction une norme de longueur sur $N$, et une norme quotient sur $\Gamma / F$, de longueur aussi.

(35) Désormais, et jusqu'au paragraphe 48, nous nous plaçons dans le cas particulier où $\Gamma$ est un sous-groupe discret cocompact d'un groupe de Lie nilpotent $G$. 
Nous notons $G_{\infty}$ le groupe gradué associé. Nous fixons une graduation

$$
\mathscr{G}_{\infty}=V_{1} \oplus \cdots \oplus V_{r}
$$

de son algèbre de Lie. Nous notons $\pi: G \rightarrow V_{1}$ l'homomorphisme composé

$$
G \rightarrow G / G^{2} \underset{\rightarrow}{\rightarrow} V_{1} \text {. }
$$

(36) D'après Malcev [12], le groupe des commutateurs $\Gamma^{2}=[\Gamma, \Gamma]$ coïncide avec $\Gamma \cap G^{2}$, l'image $\pi(\Gamma)$ est un réseau cocompact dans $V_{1}$; mieux, il existe un système générateur $\left\{\gamma_{1}^{\prime}, \ldots, \gamma_{h}^{\prime}\right\}$ de $\Gamma \cap G^{2}$, des éléments $\gamma_{1}, \ldots, \gamma_{l}$ de $\Gamma$ tels que

$$
S=\left\{\gamma_{1}^{\prime}, \ldots, \gamma_{h}^{\prime}, \gamma_{1}, \ldots, \gamma_{l}\right\}
$$

soit un système générateur de $\Gamma$, et tels que $\pi\left(\gamma_{1}\right), \ldots, \pi\left(\gamma_{l}\right)$ soit une base de $\pi(\Gamma)$. Si $w$ est la norme de mots associée à $S \cup S^{-1}$, alors la norme quotient $\bar{w}$ sur $\pi(\Gamma)$ est donnée par

$$
\bar{w}\left(\sum \lambda_{i} \pi\left(\gamma_{i}\right)\right)=\sum\left|\lambda_{i}\right| \quad \text { pour } \lambda_{i} \in \mathbb{Z} ;
$$

c'est la restriction d'une norme de l'espace vectoriel $V_{1}$.

Définition. Si | | est une norme de longueur sur $\Gamma$, la norme limite $\mid l_{\infty}$ sur $V_{1}$ correspondante est définie comme suit: sa boule unité $E$ est l'enveloppe convexe fermée des points de la forme $\pi(\gamma) /|\gamma|$, lorsque $\gamma$ décrit $\Gamma$.

Comme $\pi(\Gamma)$ est cocompact dans $V_{1}$, le convexe $E$ est d'intérieur non vide. D'après 34 , il existe une constante $c$ telle que $c|| \geq w$, d'où, pour tout $\gamma \in \Gamma$,

$$
\bar{w}(\pi \gamma) \leq c|\gamma|
$$

donc $E$ est borné; enfin, comme $|\gamma|=\left|\gamma^{-1}\right|$, le convexe $E$ est symétrique et définit bien une norme sur $V_{1}$.

(37) Nous aurons besoin au $\$ 44$ du fait que les points de la forme $\pi(\gamma) /|\gamma|$ sont denses dans la sphère unité:

Proposition. Il existe une constante $A$ et une suite $\eta_{p}$ tendant vers 0 tels que, pour tout $\alpha \in \partial E$, il existe un $\gamma \in \Gamma$ tel que

$$
p-A \leqq|\gamma| \leq p+A
$$

et

$$
|\alpha-\pi(\gamma) /| \gamma \|_{\infty} \leq \eta_{p}
$$

Démonstration. En effet, fixons un domaine fondamental $T$ de $\pi(\Gamma)$ dans $V_{1}$, et choisissons, pour chaque $\beta \in V_{1}$, un élément $\gamma(\beta)$ de $\Gamma$, de longueur minimum, tel que

$$
\beta-\pi(\gamma(\beta)) \in T \text {. }
$$

Alors

$$
\left|\pi \gamma\left(s^{\prime} \alpha\right)-\pi \gamma(s \alpha)\right|_{\infty} \leq \operatorname{diam}(T)+\left|s^{\prime}-s\right|
$$

pour tous $s, s^{\prime} \in \mathbb{R}$. Comparons les normes de $\gamma=\gamma(s \alpha)$ et de $\gamma^{\prime}=\pi\left(s^{\prime} \alpha\right)$ : si

$$
t=\bar{w}\left(\left(s^{\prime}-s\right) \alpha\right),
$$

il existe $t$ générateurs $\theta_{1}, \ldots, \theta_{t}$ tels que

$$
\pi\left(\gamma \prod \theta_{i}\right)=\pi\left(\gamma^{\prime}\right)
$$


d'où

$$
\left.\left|\gamma^{\prime}\right|<|\gamma|+t\left(\sup _{s} \mid\right)\right)
$$

Comme $\bar{w} \leq$ const. ||$_{\infty}$, nous concluons que la fonction discontinue $s \rightarrow|\gamma(s \alpha)|$ fait des sauts d'au plus const. diam $(T)=A$. Par conséquent, il existe pour tout $p$ un $\gamma=\gamma(p \alpha)$ tel que

$$
|| \gamma|-p| \leq A \quad \text { et } \quad|\pi \gamma-p \alpha|_{\infty} \leq \operatorname{diam}(T) .
$$

\section{F. Convergence de métriques sur les groupes nilpotents}

(38) Ici commence la démonstration du théorème principal. Supposons donnée une norme de longueur | | sur le sous-groupe $\Gamma$ du groupe de Lie $G$. Supposons de plus $G$ gradué, i.e. $G=G_{\infty}$ : dans ce cas, nous démontrons, dans un premier temps, que, pour tout $\gamma \in \Gamma$,

$$
\lim _{t \rightarrow \infty} \frac{1}{t}\left|\delta_{t} \gamma\right|=d_{\infty}(1, \gamma)
$$

Dans le cas général, il n'y a pas d'homothéties $\delta_{t}$ dans le groupe $G$ : nous construisons donc un espace métrique $X$ qui contient les groupes $G$ et $G_{\infty}$, et qui possède des homothéties.

(39) L'espace topologique $X$ est le produit $\left.\left.\mathscr{G}_{\infty} \times\right] 0,+\infty\right]$; définissons l'homéomorphisme $\delta_{t}: X \rightarrow X$ par la formule

$$
\left.\left.\delta_{t}(x, s)=\left(\delta_{t} x, \frac{s}{t}\right) \quad \text { pour } x \in \mathscr{G}_{\infty}, \quad s \in\right] 0,+\infty\right] .
$$

Fixons une famille de supplémentaires $W^{i}$ de $\mathscr{G}^{i+1}$ dans $\mathscr{G}^{i}$, et notons $L$ l'unique bijection linéaire de $\mathscr{G}$ sur $\mathscr{G}_{\infty}$ telle que

(i) $L\left(W^{i}\right)=V_{i}$, et

(ii) modulo les isomorphismes $W^{i} \rightarrow \mathscr{G}^{i} / \mathscr{G}^{i+1}$ et $V^{i} \Im \mathscr{G}^{i} / \mathscr{G}^{i+1}$ (définition de l'algèbre graduée associée), $L$ est l'application identique.

Munissons l'espace vectoriel

$$
\mathscr{G}_{1}=\mathscr{G}_{\infty} \times 1
$$

d'une structure d'algèbre de Lie $[,]_{1}$, de façon que

$$
L: \mathscr{G} \rightarrow \mathscr{G}_{1}
$$

soit un isomorphisme. Munissons chaque

$$
\mathscr{G}_{t}=\mathscr{G}_{\infty} \times t
$$

de la structure d'algébre de Lie $[,]_{\ell}$ telle que

$$
\delta_{1 / t}: \mathscr{G}_{1} \rightarrow \mathscr{G}_{t}
$$

soit un isomorphisme. Il résulte du choix particulier de la bijection $L$ que le crochet $[,]_{t}$ dépend continûment de $t$, pour $\left.\left.t \in\right] 0,+\infty\right]$.

Munissons aussi chaque $\mathscr{G}_{t}$ de la structure de groupe de Lie $G_{t}$ correspondante. Lorsque nous considérons $\mathscr{G}_{t}$ comme l'espace tangent à l'origine de $G_{t}$, nous utilisons l'exponentielle $\exp _{t}$ et la réciproque $\log _{t}$. L'homéomorphisme $\delta_{t}$ de $X$ se restreint 
en un isomorphisme des groupes $G_{s}$ et $G_{s / t}$; en particulier, chaque $G_{t}$ contient un exemplaire $\Gamma_{t}$ de $\Gamma$, qu'il faut imaginer comme de plus en plus dense dans $G_{t}$ lorsque $t$ tend vers $+\infty$.

(40) Pour exprimer ceci avec précision, introduisons des métriques sur $X$. Les deux sont, restreintes sur chaque groupe $G_{t}$, invariantes à gauche, et données par les longueurs des courbes.

Si $c(s)=(\sigma(s), t(s))$ est une courbe dans $X$, notons $\sigma_{t}^{\prime}(s)$ le vecteur tangent $d \sigma / d s$, ramené à l'origine par une translation à gauche de $G_{t(s)}$. On dispose, sur les espaces tangents à l'origine $\mathscr{G}_{t}$, du sous-espace $V_{1}$, qui porte la norme | lo $_{\infty}$; fixons une norme $\nu$ sur $\mathscr{G}_{\infty}$ (et donc sur les $\mathscr{G}_{t}$ ) telle que, si

$$
x=\pi x+\pi^{\prime} x,
$$

où $\pi x \in V_{1}, \pi^{\prime} x \in V_{2} \oplus \cdots \oplus V_{n}$ alors

$$
\nu(x)=|\pi x|_{\infty}+\nu\left(\pi^{\prime} x\right) .
$$

La distance $D$ sur $X$ est donnée par

$$
\operatorname{longueur}_{D}(c)=\int_{0}^{1} \nu\left(\sigma_{t}^{\prime}(s)\right)+\left|\left(\frac{1}{t}\right)^{\prime}(s)\right| d s
$$

la distance $Q$ sur $X$ est donnée par

$$
\operatorname{longueur}_{Q}(c)=\int_{0}^{1}\left|\sigma_{t}^{\prime}(s)\right|_{\infty}+\left|\left(\frac{1}{t}\right)^{\prime}(s)\right| d s ;
$$

si $\sigma_{t}^{\prime}(s)$ est presque toujours tangent à $\dot{V_{1}}$, longueur ${ }_{Q}(c)=+\infty$ sinon.

Les $\delta_{t}$ sont des homothéties pour la distance $Q$; d'après le théorème de Süssmann $(\S 20)$, les deux distances sont compatibles avec la topologie de $X$.

(41) Proposition. Si les $\gamma_{t} \in \Gamma_{t}$ convergent vers $x \in \mathscr{G}_{\infty}$ alors

$$
\lim _{t \rightarrow+\infty} \frac{1}{t}\left|\gamma_{t}\right|=d_{\infty}(1, x)
$$

La démonstration de cette proposition procède en trois étapes.

D'abord (\$44), nous montrons que, pour tout $x \in G_{\infty}$, il existe une suite $\gamma_{t} \in \Gamma_{t}$ telle que

$$
\lim \gamma_{t}=x \quad \text { et } \quad \lim \frac{1}{t}\left|y_{t}\right| \leq d_{\infty}(1, x) .
$$

Pour cela, étant donnée une courbe $c(s)$ dans $G_{\infty}$, tangente à $V_{1}$, reliant l'origine 1 à $x$, nous approchons chaque vecteur unitaire $c^{\prime}(s)$ par un vecteur de la forme $\pi(\gamma) /|\gamma|$, donc l'élément de courbe

$$
c(s)^{-1} c(s+d s)
$$

par l'élément $\delta_{d s}(\gamma) \in \Gamma_{1 / d s}$. Pour $t=1 / d s$, l'élément

$$
\gamma_{t}=\prod_{k=1}^{t} \delta_{d s} \gamma(k d s)
$$

tend vers $x$. 
Ensuite ( $\$ 45$ ), nous montrons que, si $\gamma_{t}, \gamma_{t}^{\prime} \in \Gamma_{t}$ ont même limite $x$, alors

$$
\frac{1}{t}\left(\left|\gamma_{t}\right|-\left|\gamma_{t}^{\prime}\right|\right)
$$

tend vers 0 .

Enfin ( $\$ 46)$, au moyen de la propriété de longueur, nous relions 1 à $\gamma_{t}$ dans $\Gamma_{t}$ par une chaîne d'éléments de ce groupe, tels que la courbe linéaire par morceaux $c_{t}(s)$ qui passe par ces points ait une vitesse uniformément bornée pour la métrique $D$. Par le théorème d'Ascoli, une sous-suite converge uniformément (pour $D$ ) vers une courbe $c_{\infty}$ dans $G_{\infty}$; comme les $c_{t}$ sont presque horizontales, on a, d'après le lemme 18 ,

$$
\nu\left(\pi^{\prime} \log _{t} c_{t}(s)^{-1} c_{t}(s+\varepsilon)\right) \leq \text { const. } \varepsilon^{2}
$$

par passage à la limite, nous concluons que $c_{\infty}$ est presque partout tangente à $V_{1}$, et donc que

$$
\begin{aligned}
d_{\infty}(1, x) & \leq \operatorname{long}_{\infty}\left(c_{\infty}\right)=\operatorname{long}_{D}\left(c_{\infty}\right) \\
& \leq \liminf _{t \rightarrow+\infty} \operatorname{long}_{D}\left(c_{t}\right)=\liminf _{t \rightarrow+\infty} \frac{1}{t}\left|\gamma_{t}\right| .
\end{aligned}
$$

Nous aurons besoin de deux lemmes, l'un est un raffinement du lemme 18, l'autre un théorème des accroissements finis pour un groupe de Lie.

(42) Lemme. Soit $\gamma \in \Gamma=\Gamma_{1}$. Pour $t$ assez petit, on a

$$
\nu\left(\pi^{\prime} \log _{1 / t} \delta_{t} \gamma\right) \leq \text { const. } t^{2}|\gamma|^{2} .
$$

Démonstration. Estimons d'abord à quel point l'exponentielle, dans le groupe $G_{s}$, n'est pas un homomorphisme: de la formule de Campbell \& Hausdorff, nous tirons l'inégalité, pour $X, Y \in \mathscr{G}_{s}$

$$
\nu(\log (\exp X \exp Y)-X-Y) \leq C_{s} \nu(X) \nu(Y),
$$

où $C_{s}$ ne dépend que de la norme du crochet $[,]_{s}$, application bilinéaire sur l'espace normé $\mathscr{G}_{s}$ muni de $\nu$. Par récurrence, il vient, pour des $Y_{i}$ assez petits,

$$
\nu\left(\log \prod \exp Y_{i}-\sum Y_{i}\right) \leq C_{s} \sigma_{2}\left(\nu\left(Y_{i}\right)\right)+\cdots+C_{s}^{p-1} \sigma_{p}\left(\nu\left(Y_{i}\right)\right)
$$

où les $\sigma_{i}$ sont les fonctions symétriques élémentaires. Le second membre s'écrit aussi

$$
C_{s}^{-1}\left\{\prod\left(1+C_{s} \nu\left(Y_{i}\right)\right)-C_{s} \sum \nu\left(Y_{i}\right)-1\right\}
$$

Posons $u=\sum \nu\left(Y_{i}\right)$; si $u$ est petit, on a

$$
C_{s}^{-1}\left(\exp \left(C_{s} u\right)-C_{s} u-1\right) \leq \text { const. } C_{s} u^{2} \text {, }
$$

d'où enfin, pour un nombre quelconque de vecteurs $Y_{i} \in \mathscr{G}_{s}$, pourvu que $u=\sum \nu\left(Y_{i}\right)$ soit assez petit:

$$
\nu\left(\log \prod \exp Y_{i}-\sum Y_{i}\right) \leqq \text { const. } C_{s} u^{2} .
$$

Soit $w$ une norme de mots sur $\Gamma$ telle que $w \leqq c||$.

Fixons un $\gamma \in \Gamma$, notons $p=w(\gamma)$ : il existe $p$ générateurs $\theta_{1}, \ldots, \theta_{p}$ tels que

$$
\gamma=\prod \theta_{i}, \quad \text { et } \quad \nu\left(\log _{1} \theta_{i}\right) \leqq c^{\prime}
$$


Alors

$$
\nu\left(\log _{1 / t}\left(\delta_{t} \gamma\right)-\sum \log _{1 / t} \delta_{t} \theta_{i}\right) \leq \text { const. } C_{1 / t} u^{2}
$$

où

$$
u=\sum \nu\left(\log _{1 / t} \delta_{t} \theta_{i}\right)
$$

Or

$$
\log _{1 / t} \delta_{t} \theta_{i}=\delta_{t} \log _{1} \theta_{i} \sim t \pi\left(\theta_{i}\right)
$$

donc

$$
\nu\left(\log _{1 / t} \delta_{t} \theta_{i}\right) \leq c^{\prime} t \quad \text { et } \quad u \leq c^{\prime} p t .
$$

D'autre part, écrivons $\log _{1} \theta_{i}=z_{1}+\cdots+z_{r}$; alors

$$
\pi^{\prime}\left(\delta_{t} \log _{1} \theta_{i}\right)=t^{2} z_{2}+\cdots+t^{\prime} z_{r}
$$

donc sa norme est majorée par $c^{\prime} t^{2}$ si $t$ est petit. Nous concluons que

$$
\begin{aligned}
\nu\left(\log _{1 / t} \delta_{t} \gamma\right) & \leq \text { const. } C_{1 / t} p^{2} t^{2}+\nu\left(\sum \pi^{\prime} \delta_{t} \log _{1} \theta_{i}\right) \\
& \leq \text { const. } C_{1 / t} p^{2} t^{2} \\
& \leq \text { const. }|\gamma|^{2} t^{2}
\end{aligned}
$$

car les normes des crochets $[,]_{s}$ sont bornées lorsque $s$ tend vers l'infini. En effet, à cause du choix particulier de la bijection linéaire

$$
L: \mathscr{G} \rightarrow \mathscr{G}_{\infty},
$$

les applications bilinéaires $[,]_{s}$ sur $\mathscr{G}_{\infty}$ convergent vers $[,]_{\infty}$.

(43) LEMME. Soient, dans le groupe $G_{t}$, deux arcs $c_{1}, c_{2}:[0,1] \rightarrow G_{t}$, de même origine 1 , de vitesse inférieure à $K$. Notons $c_{i}^{\prime}(s)$ le vecteur vitesse $d c_{i} / d s$ ramené à l'origine par une translation à gauche. Si, pour tout $s$,

alors

$$
\nu\left(c_{1}^{\prime}(s)-c_{2}^{\prime}(s)\right) \leqq F,
$$

Démonstration. Posons $f(t)=D\left(c_{1}(t), c_{2}(t)\right)$. Il vient

$$
\begin{aligned}
|f(s)-f(t)| & \leq\left|D\left(c_{1}(s), c_{2}(s)\right)-D\left(c_{1}(s), c_{1}(s) c_{1}(t)^{-1} c_{2}(t)\right)\right| \\
& \leq D\left(c_{2}(s), c_{1}(s) c_{1}(t)^{-1} c_{2}(t)\right)
\end{aligned}
$$

posons

$$
a=c_{1}(s)^{-1} c_{2}(s), \quad X=c_{1}^{\prime}(s), \quad Y=c_{2}^{\prime}(s) ;
$$

alors le second membre est de l'ordre de

$$
\nu \log \left(a^{-1} \exp (s-t) X a \exp (t-s) Y\right)
$$

or

donc

$$
\begin{aligned}
a^{-1} \exp (s-t) X a \exp (t-s) Y & \sim \exp (s-t) \operatorname{Ad}_{a} X \exp (t-s) Y \\
& \sim \exp (t-s)\left(Y-\operatorname{Ad}_{a} X\right)
\end{aligned}
$$

$$
\left|f^{\prime}(s)\right| \leq \nu\left(Y-\operatorname{Ad}_{a} X\right) \leq \nu(Y-X)+\left\|\operatorname{Ad}_{a}-\operatorname{Id}\right\| K .
$$


Il reste à majorer la norme de l'endomorphisme $\mathrm{Ad}_{a}-\mathrm{Id}$ : posons

$$
a=\exp A \text {; }
$$

alors

$$
\operatorname{Ad}_{a}=\exp (\operatorname{ad} A)
$$

d'où

$$
\left\|\mathrm{Ad}_{a}-\mathrm{Id}\right\| \leq \exp (\|\operatorname{ad} A\|)-1 \leq \exp \left(C_{l} D(1, a)\right)-1
$$

comme

$$
D(1, a)(=f(s)) \leq D\left(1, c_{1}(s)\right)+D\left(1, c_{2}(s)\right) \leq 2 K,
$$

on a

$$
\exp \left(C_{t} D(1, a)\right) \leq 1+\text { const }(K) C_{t} D(1, a),
$$

d'où enfin l'inéquation différentielle

$$
f^{\prime}(s) \leq F+\text { const }(K) f(s) .
$$

Avec la condition initiale $f(0)=0$, il vient

$$
f(1) \leq F \text { const }(K) \text {. }
$$

car on peut majorer $C_{t}$ independamment de $t$.

(44) Soit $x \in G_{\infty}$; il existe une famille $t \mapsto \gamma_{t} \in \Gamma_{t}$ telle que $\gamma_{t}$ tende vers $x$ lorsque $t$ tend vers $+\infty$, et que

$$
\limsup _{t \rightarrow+\infty} \frac{1}{t}\left|\gamma_{t}\right| \leq d_{\infty}(1, x)
$$

Fixons une courbe lisse $c:[0,1] \rightarrow G_{\infty}$, tangente à $V_{1}$, reliant 1 à $x$, de longueur à peine supérieure à $d_{\infty}(1, x)$. Par commodité, posons long $g_{\infty}(c)=1$, et paramétrisons $c$ de façon que $\left|c^{\prime}(s)\right|_{\infty}=1$. Fixons un entier $N$ et un $p>0$; approchons $c$ par la courbe horizontale, linéaire par morceaux $\bar{c}$, donnée par:

$$
\bar{c}(s)=\bar{c}\left(\frac{k}{N}\right) \exp \left(\left(s \frac{-k}{N}\right) c^{\prime}\left(\frac{k}{N}\right)\right), \quad \text { pour } s \in\left[\frac{k}{N}, \frac{k+1}{N}[.\right.
$$

Comme $N$, qui dépendra de $t$, tendra vers $+\infty$, il suffit de construire $\gamma_{t}$ qui approche $\bar{c}(1)$. D'après la proposition 37, il existe, pour tout $k=1, \ldots, N$ un $\gamma^{k} \in \Gamma$, tel que

$$
|| \gamma^{k}|-p| \leq A \quad \text { et } \quad\left|c^{\prime}\left(\frac{k}{N}\right)-\pi\left(\gamma^{k}\right) /\right| \gamma k \| \leq \eta_{p}
$$

Posons

$$
\gamma_{t}=\prod \delta_{1 / t} \gamma^{k}
$$

alors

$$
\left|\gamma_{t}\right| \leq N(p+A)
$$

Construisons, en utilisant les $\gamma^{k}$ comme vitesses successives, le chemin linéaire par morceaux $c_{t}$ dans $G_{t}$ : on a $c_{t}(0)=1$, et si $s \in[(k / N),(k+1) / N[$,

$$
c_{t}(s)=c_{t}\left(\frac{k}{N}\right) \exp _{t}(N s-k) \log _{t} \delta_{1 / t} \gamma^{k}
$$


Comparons le à $\bar{c}_{t}$ :

$$
\bar{c}_{t}(s)=\bar{c}_{t}\left(\frac{k}{N}\right) \exp _{t}(N s-k) \frac{1}{N} c^{\prime}\left(\frac{k}{N}\right)
$$

d'après le lemme 42,

$$
\nu\left(\log _{t} \delta_{1 / t} \gamma^{k}-\frac{1}{t} \pi \gamma^{k}\right) \leq \text { const. } p^{2} t^{-2}
$$

si $N t^{-1} p$ tend vers 1 , alors $\nu\left(c_{t}^{\prime}(s)-N t^{-1} \pi\left(\gamma^{k}\right)\right)$ tend vers 0 ; mais aussi

$$
\nu\left(N t^{-1} \pi\left(\gamma^{k}\right)-\pi\left(\gamma^{k}\right) /\left|\gamma^{k}\right|\right) \text { et } \nu\left(\pi\left(\gamma^{k}\right) /\left|\gamma^{k}\right|-\bar{c}_{t}(s)\right)
$$

si de plus $p$ tend vers l'infini. Par conséquent, $\sup \nu\left(c_{t}^{\prime}(s)-\bar{c}_{t}^{\prime}(s)\right)$ tend vers 0 lorsque $p, t$ tendent vers $+\infty$, et $N p t^{-1}$ vers 1 ; d'après le lemme 43,

$$
D\left(\gamma_{t}, \bar{c}_{t}(1)\right)=D\left(c_{t}(1), \bar{c}_{t}(1)\right)
$$

tend vers 0 .

Enfin, l'application

$$
\frac{1}{t} \mapsto \log _{t} \bar{c}_{t}(1), \quad \mathbb{R} \rightarrow \mathscr{G}_{\infty},
$$

est polynomiale, donc $D\left(\bar{c}(1), \bar{c}_{t}(1)\right)$ tend vers 0 . Ceci prouve que $\gamma_{t}$ tend vers $x$; par construction,

$$
\lim \sup \left|\gamma_{t}\right| / t \leq \lim \sup N t^{-1}(p+A) \leq 1
$$

(45) Pour toute suite $\gamma_{n} \in \Gamma_{t_{n}}$ tendant vers $x \in G$, on a

$$
\limsup _{n \rightarrow+\infty} t_{n}^{-1}\left|\gamma_{n}\right| \leq d_{\infty}(1, x) \text {. }
$$

Si $\gamma_{t}$ est la famille fournie par le $\S 44$, posons

$$
\gamma_{n}^{\prime}=\gamma_{t_{n}}^{-1} \gamma_{n} \in \Gamma_{t_{n}}
$$

alors $\gamma_{n}^{\prime}$ tend vers $1 \in G_{\infty}$, donc $Q\left(1, \gamma_{n}^{\prime}\right)$ tend vers 0 . Or la restriction de $Q$ à $\Gamma_{1}$ est une norme de longueur donc il existe un constante $c$ telle que

$$
Q\left(1, \delta_{t_{n}} \gamma_{n}^{\prime}\right) \geq c\left|\gamma_{n}^{\prime}\right| \text {. }
$$

Il s'ensuit que $t_{n}^{-1}\left|\gamma_{n}^{-1} \gamma_{n}^{\prime}\right| \leq c^{-1} Q\left(1, \gamma_{n}^{\prime}\right)$ tend vers 0 , d'où

$$
\lim \sup t_{n}^{-1}\left|\gamma_{n}\right| \leq \lim \sup t_{n}^{-1}\left|\gamma_{t_{n}}\right| \leq d_{\infty}(1, x)
$$

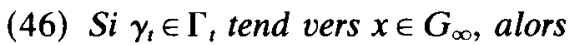

$$
\liminf _{t \rightarrow+\infty} \frac{1}{t}\left|\gamma_{t}\right| \geq d_{\infty}(1, x)
$$

Fixons une sous-suite $\gamma_{t_{n}}$ telle que $t_{n}^{-1}\left|\gamma_{t_{n}}\right|$ tende vers la limite inférieure-l'indice $n$ sera sous-entendu dans les calculs. On peut supposer que cette limite est finie.

Fixons un $\varepsilon>0$. D'après la propriété de longueur, il existe un $p$ tel que, pour tout $t$, on puisse écrire

et

$$
\begin{gathered}
\gamma_{t}=\prod \gamma_{t}^{k}, \quad \text { où } \quad \gamma_{t}^{k} \in \Gamma_{t}, \quad\left|\gamma_{t}^{k}\right| \leq p, \\
\sigma=\sum \gamma_{t}^{k} \leq(1+\varepsilon)\left|\gamma_{t}\right| .
\end{gathered}
$$

Posons $s_{k}=\sum^{k-1}\left|\gamma_{t}^{j}\right| / \sigma$, et construisons l'arc linéaire par morceaux

$$
c_{t}(s)=c_{t}\left(s_{k}\right) \exp _{t}\left[\left(s-s_{k}\right) /\left(s_{k+1}-s_{k}\right)\right] \log _{t} \gamma_{t}^{k} .
$$


Son vecteur vitesse, ramené à l'origine, est

$$
\left(\log _{t} \gamma_{t}^{k}\right) \sigma /\left|\gamma_{t}^{k}\right|
$$

Montrons qu'il est borné indépendamment de $t$ : d'après le lemme 42,

$$
\nu\left(\log _{t} \gamma_{t}^{k}-\frac{1}{t} \pi \gamma_{t}^{k}\right) \leq \text { const. } t^{-2}\left|\gamma_{t}^{k}\right|^{2},
$$

d'où

$$
\nu\left(c_{t}^{\prime}(s)\right) \leq \sigma / t+\text { const. } \sigma p t^{-2}
$$

comme

$$
\sigma / t \leq(1+\varepsilon) \frac{1}{t}\left|\gamma_{t}\right|
$$

est borné lorsque $t$ tend vers 0 , les applications $c_{t}:[0,1] \rightarrow X$ sont équicontinues. Nous pouvons donc supposer qu'elles convergent uniformément vers $c_{\infty}:[0,1] \rightarrow G_{\infty}$, pour la distance $D$. Par semi-continuité de la longueur (la métrique $D$ est finslérienne), on a

$$
\operatorname{long}_{D}\left(c_{\infty}\right) \leq \lim \operatorname{long}_{D}\left(c_{t}\right) \leq(1+\varepsilon) \lim \frac{1}{t}\left|\gamma_{t}\right|
$$

Il ne reste plus qu'à vérifier que $c$ est presque partout tangente à $V_{1}$.

Remarquons que le pas des subdivisions $s_{k}$ tend vers 0 si $x \neq 1$; en effet, dans ce cas,

$$
s_{k+1}-s_{k} \leq p / \sigma
$$

et

$$
\sigma / t \geq\left|\gamma_{t}\right| / t \geq \text { const. } Q\left(1, \gamma_{t}\right)
$$

qui ne tend pas vers 0 .

Etant donnés $s \in[0,1], \eta$ petit, on peut donc écrire

$$
s=\lim _{t \rightarrow+\infty} s_{i}, \quad s+\eta=\lim _{t \rightarrow+\infty} s_{j}
$$

d'où

$$
\pi^{\prime}\left(\log _{\infty} c_{\infty}(s)^{-1} c_{\infty}(s+\eta)\right)=\lim \pi^{\prime}\left(\log _{t} \prod_{k=i}^{j-1} \gamma_{t}^{k}\right)
$$

or, d'après le lemme 42 ,

$$
\nu\left(\pi^{\prime} \log _{t} \Pi \gamma_{t}^{k}\right) \leq \text { const. } t^{-2}\left|\prod \gamma_{t}^{k}\right|^{2}
$$

on a

$$
\left|\Pi \gamma_{t}^{k}\right| \leq \sum\left|\gamma_{t}^{k}\right|=\sigma\left(s_{j}-s_{i}\right) \leq \text { const. } t \eta
$$

d'où

$$
\nu\left(\pi^{\prime} \log _{\iota} \prod_{k=i}^{j-1} \gamma_{t}^{k}\right) \leq \text { const. } \eta^{2}
$$

ceci prouve que, pour chaque $s$ tel que $c_{\infty}$ soit dérivable en $s$ (i.e. presque partout, car $c_{\infty}$ est Lipschitz), la dérivée $d c / d s$ est tangente à $V_{1}$. 
Ceci achève la démonstration de la proposition 41 . Le même argument donne aussi la

(47) Proposition. Si $\gamma_{t}, \gamma_{t}^{\prime} \in \Gamma_{t}$, si $\gamma_{t}$ tend vers $x \in G_{\infty}$, si $\gamma_{t}^{\prime}$ tend vers $x^{\prime} \in G_{\infty}$ pour la topologie de $X$, alors

$$
\lim _{t \rightarrow+\infty} \frac{1}{t}\left|\gamma_{t}^{-1} \gamma_{t}^{\prime}\right|=d_{\infty}\left(x, x^{\prime}\right)
$$

(48) La proposition 41 signifie que, lorsque de grandes distances sont en jeu, on peut remplacer la norme || donnée sur $\Gamma$ par la distance de Carnot-Carathéodory.

En effet, notons $\left.\left.h: X=G_{\infty} \times\right] 0,+\infty\right] \rightarrow G_{\infty}$ la projection sur le premier facteur. En particulier, $\left.h\right|_{G_{1}}$ est obtenu en composant $\log _{1}$, $\exp _{\infty}$ et la bijection linéaire $L$ : $\mathscr{G} \rightarrow \mathscr{G}_{\infty}$. Cet homéomorphisme $h$ de $G$ sur le gradué associé $G_{\infty}$ dépend donc du choix d'une graduation de $\mathscr{G}_{\infty}$ et d'une famille de supplémentaires $W_{1}$ de $\mathscr{G}^{i+1}$ dans $\mathscr{G}^{i}$

COROllaire. Lorsque $\gamma \in \Gamma$ tend vers l'infini, le rapport

$$
d_{\infty}(1, h(\gamma)) /|\gamma|
$$

tend vers 1 .

Démonstration. Si le corollaire est en défaut, il existe une suite $\gamma_{n}$ dans $\Gamma$ telle que $\left|\gamma_{n}\right|$ tende vers l'infini, alors que, si

$$
t_{n}=d_{\infty}\left(1, h \gamma_{n}\right),
$$

le rapport $\left|\gamma_{n}\right| / t_{n}$ ne tende pas vers 1 . Posons

$$
x_{n}=\delta_{1 / t_{n}} \gamma_{n} \in G_{t_{n}}
$$

alors $d_{\infty}\left(1, h x_{n}\right)=1$, donc nous pouvons supposer que $x_{n}$ converge vers un $x \in G_{\infty}$ tel que $d_{\infty}(1, x)=1$. La proposition 41 entraîne que $\left(1 / t_{n}\right)\left|\gamma_{n}\right|$ tend vers $d_{\infty}(1, x)=1$, ce qui contredit l'hypothèse.

(49) Démonstration du théorème principal. [Si | est une norme de longueur sur le groupe discret $\Gamma$, de type fini, presque nilpotent, alors les espaces $(\Gamma, \varepsilon||)$ convergent au sens de Hausdorff pointé, lorsque $\varepsilon$ tend vers 0 , vers l'espace $\left(G_{\infty}, d_{\infty}\right)$ (décrit aux $\S 13$ à § 25).]

Supposons d'abord $\Gamma$ cocompact dans un groupe de Lie nilpotent, simplement connexe, $G$. Utilisant le critère des résaux $(\$ 8)$, nous montrons que, pour tout $R>0$, il existe un fonction $\theta(\varepsilon)$ tendant vers 0 telle que la boule de rayon $R+\theta(\varepsilon)$ dans $(\Gamma, \varepsilon||)$ converge au sens de Hausdorff vers la boule de rayon $R$ dans $\left(G_{\infty}, d_{\infty}\right)$, notée $B_{\infty}(R)$.

Pour $r>0$, notons

$$
\Gamma^{r}=\{\gamma:|\gamma| \leq r\} \subset \Gamma
$$

notons $C_{r}$ le cylindre

$$
C_{r}=B_{\infty}(r) \times[1,+\infty] \subset X,
$$

fixons un domaine fondamental $P$ pour l'action de $\Gamma$ dans $G$. Comme $\left(C_{R}, Q\right)$ est compact, il existe, pour tout $\eta>0$, un ensemble fini $N_{\eta} \subset B_{\infty}(R)$ tel que, relativement 
à la distance $Q, C_{R}$ soit contenu dans le $\eta$-voisinage de $N_{\eta} \times[1,+\infty]$. Pour $x \in N_{\eta}$, notons $x_{\varepsilon}$ l'élément $\left(\log _{\infty} x, 1 / \varepsilon\right)$ de $G_{1 / \varepsilon}$.

Pour $x \in N_{\eta}$, notons $h_{\varepsilon}(x)$ l'élément $\gamma$ de $\Gamma$ tel que $\delta_{1 / \varepsilon} x_{\varepsilon} \in \gamma P$. Alors les $\delta_{\varepsilon} h_{\varepsilon}(x)$ convergent vers $x \in G_{\infty}$ : en effet,

$$
Q\left(\delta_{\varepsilon} h_{\varepsilon}(x), x_{\varepsilon}\right)=\varepsilon Q\left(h_{\varepsilon}(x), \delta_{1 / \varepsilon} x_{\varepsilon}\right) \leq \varepsilon \operatorname{Diam}_{O}(P)
$$

tend vers 0 avec $\varepsilon$. Par conséquent, d'après la proposition 41 ,

$$
\varepsilon\left|h_{\varepsilon}(x)\right| / d_{\infty}(1, x)
$$

tend vers 1 , donc l'image $h_{\varepsilon}\left(N_{\eta}\right)$ est contenue dans une boule $\Gamma^{(R+\theta) / \varepsilon}$, où $\theta(\varepsilon)$ tend vers 0 avec $\varepsilon$. De plus, si $x \neq x^{\prime} \in N_{\eta}$, le rapport

$$
\varepsilon\left|h_{\varepsilon}(x)^{-1} h_{\varepsilon}\left(x^{\prime}\right)\right| / d_{\infty}\left(x, x^{\prime}\right)
$$

tend vers 1 , donc $h_{\varepsilon}$ est presque une isométrie de $N_{\eta}$ sur une partie de $\Gamma^{(R+\theta) / \varepsilon}$.

Il reste à montrer que cette partie est très dense dans $\Gamma^{(R+\theta) / \varepsilon}$, i.e. que pour tout $\gamma \in \Gamma^{(R+\theta) / \varepsilon}$, il existe un $x \in N_{\eta}$ tel que $\varepsilon\left|\gamma^{-1} h_{\varepsilon}(x)\right| \leq$ const. $\eta$. D'après le corollaire 48 , on a, pour tout $\gamma \in \Gamma^{(R+\theta) / \varepsilon}$,

$$
d_{\infty}(1, h \gamma) \leq \frac{R+\theta^{\prime}}{\varepsilon}
$$

pour un $\theta^{\prime}(\varepsilon)$ tendent vers 0 avec $\varepsilon$. Par conséquent,

$$
d_{\infty}\left(1, h \delta_{\varepsilon} \gamma\right)=d_{\infty}\left(1, \delta_{\varepsilon} h \gamma\right)=\varepsilon d_{\infty}(1, h \gamma) \leq R+\theta^{\prime}
$$

donc il existe un $z \in C_{R}$ tel que

$$
d_{\infty}\left(h z, h \delta_{\varepsilon} \gamma\right) \leq \theta^{\prime}
$$

d'où

$$
Q\left(z, \delta_{\varepsilon} \gamma\right) \leq \theta^{\prime \prime},
$$

où $\theta^{\prime \prime}(\varepsilon)$ tend vers 0 avec $\varepsilon$. Enfin, il existe un $x \in N_{\eta}$ tel que

$$
Q\left(x_{\varepsilon}, \delta_{\varepsilon} \gamma\right) \leq \eta+\theta^{\prime \prime}
$$

soit

$$
Q\left(\delta_{1 / \varepsilon} x_{\varepsilon}, \gamma\right) \leq\left(\eta+\theta^{\prime \prime}\right) / \varepsilon
$$

et donc

$$
Q\left(h_{\varepsilon}(x), \gamma\right) \leq \frac{\eta+\theta^{\prime \prime}}{\varepsilon}+\operatorname{diam}_{Q} P .
$$

Comme $\left.Q\right|_{\Gamma}$ et || sont deux normes de longueur sur $\Gamma$, nous concluons que

$$
\varepsilon\left|\gamma^{-1} h_{\varepsilon}(x)\right| \leq \text { const. }\left(\eta+\theta^{\prime \prime}\right)+\varepsilon \text { diam }_{Q} P \leq \text { const.' } \eta
$$

lorsque $\varepsilon$ est petit.

Cas général. Soit $N$ un sous-groupe nilpotent, d'indice fini dans $\Gamma$. Les éléments de torsion forment un sous-groupe fini $F$ dans $N$ (voir l'appendice). Le groupe quotient $\Gamma^{\prime}, \Gamma^{\prime}=N / F$, de type fini, nilpotent, sans torsion, est, d'après Malcev [12], isomorphe à un sous-groupe cocompact d'un groupe de Lie nilpotent simplement connexe $G$. Munissons $\Gamma^{\prime}$ de la norme quotient; alors la distance de Hausdorff (non pointée) de $\Gamma$ à $\Gamma^{\prime}$ est finie, donc la convergence des $(\Gamma, \varepsilon||)$ résulte de celle des homothétiques de $\Gamma^{\prime}$. 
(50) Achevons cette partie par un lemme technique.

LEMME. Soit $P$ un domaine fondamental pour l'action de $\Gamma$ dans $G$. Notons $h$ la projection sur le premier facteur de $X, h: G \rightarrow G_{\infty}$. Posons, pour $r>0$,

$$
\Delta(r)=\sup \left\{\operatorname{diam}_{\infty}(h(\gamma P)): d_{\infty}(1, h \gamma)<r\right\}
$$

Alors $\Delta(r) / r$ tend vers 0 lorsque $r$ tend vers $+\infty$.

Démonstration. On peut écrire

$$
\Delta(r)=d_{\circ}\left(h \gamma_{r} x_{r}, h \gamma_{r} y_{r}\right)
$$

où $x_{r}, y_{r} \in P, \gamma_{r} \in \Gamma, d_{\infty}\left(1, h \gamma_{r}\right) \leq r, \gamma_{r}$ tend vers l'infini. Posons

$$
a_{r}=\delta_{1 / r} \gamma_{r} x_{r} \in G_{r}, \quad b_{r}=\delta_{1 / r} \gamma_{r} y_{r}
$$

Les suites $a_{r}$ et $b_{r}$ sont bornées dans $X$ : en effet,

$$
Q\left(1, a_{r}\right)=Q\left(1, \gamma_{r} x_{r}\right) / r \leq Q\left(1, \gamma_{r}\right) / r+\operatorname{diam}_{Q} P / r
$$

or

$$
Q\left(1, \gamma_{r}\right) \leq \text { const. }\left|\gamma_{r}\right| \leq \text { const' }^{\prime} . d_{\infty}\left(1, h \gamma_{r}\right) .
$$

Comme $Q$ est invariante à gauche sur $G=G_{1}$,

$$
Q\left(a_{r}, b_{r}\right)=Q\left(x_{y}, y_{r}\right) / r \leq \operatorname{diam}_{Q} P / r
$$

tend vers 0 . La distance $Q$ et la distance produit ' $d_{\infty} \oplus d(1 / t)$ étant uniformément équivalentes sur les parties bornées de $X$, on conclut que

$$
\Delta(r) / r=d_{\infty}\left(h a_{r}, h b_{r}\right)
$$

tend vers 0 .

G. Volume des boules

(51) Soit $(M, g)$ une variété riemannienne compacte, dont le groupe fondamental est presque nilpotent. Rappelons que $\operatorname{vol}_{\tilde{m}}(R)$ désigne le volume de la boule de centre $\tilde{m}$, de rayon $R$ dans le revêtement universel $(\tilde{M}, \tilde{g})$.

Proposition. Si p est la dimension homogène du groupe fondamental $\Gamma$, la limite

$$
\lim _{R \rightarrow \infty} \operatorname{vol}_{\tilde{m}}(R) R^{-p},
$$

où $p$ est la dimension homogène du groupe $\Gamma$, existe toujours.

Démonstration. Soit $\tilde{P}$ un domaine fondamental pour l'action de $\Gamma$ sur $\tilde{M}$; notons $\delta=\operatorname{diam}_{\tilde{g}} \tilde{P}$. On a alors les inclusions

$$
\bigcup_{|\gamma| \leq R-\delta} \gamma \tilde{P} \subset B(\tilde{m}, R) \subset \bigcup_{|\gamma| \leq R+\delta} \gamma \tilde{P}
$$

d'où

$$
\lim _{R \rightarrow \infty} \operatorname{vol}(g) \operatorname{card}\{\gamma:|\gamma| \leq R\} / \operatorname{vol}_{\tilde{m}}(R)=1 .
$$

Cas où $\Gamma$ est isomorphe à un sous-groupe discret cocompact d'un groupe de Lie nilpotent $G$. Clairement, il résulte du corollaire 48 que le rapport

$$
\operatorname{card}\{\gamma:|\gamma| \leq R\} / \operatorname{card}\left\{\gamma: d_{\infty}(1, h \gamma) \leq R\right\}
$$


tend vers 1 lorsque $R$ tend vers $+\infty$. Fixons un domaine fondamental $P$ pour $\Gamma$ dans $G$; avec les notations du lemme 50 ,

$$
\bigcup\left\{h(\gamma P): d_{\infty}(1, h \gamma) \leq R-\Delta(R)\right\} \subset B_{\infty}(R) \subset \bigcup\left\{h(\gamma P): d_{\infty}(1, h \gamma) \leq R+\Delta(R)\right\} .
$$

Pour toute mesure de Haar $\mu$ sur $G_{\infty}$, la mesure induite $h^{*} \mu$ est une mesure de Haar sur $G$, qui ne dépend pas des choix qui interviennent dans la définition de $h$. Par conséquent, $\mu h(\gamma P)$ ne dépend pas de $\gamma$, et il vient

$$
\lim _{R \rightarrow \infty} \mu(h P) \operatorname{card}\left\{\gamma: d_{\infty}(1, h \gamma) \leq R\right\} / \mu B_{\infty}(R)=1 .
$$

La proposition résulte alors de l'homogénéité des mesures de Haar de $G_{\infty}$ par rapport aux homothéties $\delta_{t}$ :

$$
\mu B_{\infty}(R)=R^{p} \mu B_{\infty}(1) .
$$

Le volume asymptotique est donc égal, dans ce cas, à

$$
\operatorname{vol}(g) \mu B_{\infty}(1) / \mu(h P) \text {. }
$$

Cas général. Quitte à prendre un revêtement fini (ce qui ne change pas le revêtement universel), on peut supposer que $\Gamma$ est nilpotent; notons $F$ le sous-groupe de torsion, $\Gamma^{\prime}=\Gamma / F$ muni de la norme quotient; en fonction de

$$
n(R)=\operatorname{card}\{\gamma \in \Gamma:|\gamma| \leq R\}
$$

on a les inégalités

$$
n(R) \leq n^{\prime}(R) \text { card }(F) \leq n^{\prime}(R+\sup \{|\tau|: \tau \in F\}),
$$

donc la convergence de $n(R) R^{-p}$ résulte de celle de $n^{\prime}(R) R^{-p}$.

(52) Remarque. Bien que, dans les parties $E$ et $F$, nous supposions toujours donnés un sous-groupe cocompact d'un groupe de Lie nilpotent et une métrique invariante sur ce sous-groupe discret, les démonstrations du théorème principal et de la prop. 51 s'appliquent au cas d'un groupe de Lie nilpotent muni d'une métrique invariante à gauche, même si celui-ci n'admet pas de réseau.

\section{H. Volume Asymptotique en dimension 3}

(53) Une algèbre de Lie nilpotente, non abélienne, de dimension 3 , admet nécessairement la graduation $V_{1} \oplus \mathscr{G}^{2}$, où $\operatorname{dim} V_{1}=2, \operatorname{dim} \mathscr{G}^{2}=1$; elle est donc unique à isomorphisme près. D'après [12], tout sous-groupe discret cocompact de $G$ admet des générateurs $\gamma_{1}, \gamma_{2}, \gamma_{3}$ satisfaisant aux relations

$$
\left[\gamma_{1}, \gamma_{2}\right]=\gamma_{3}^{k}, \quad\left[\gamma_{1}, \gamma_{3}\right]=\left[\gamma_{2}, \gamma_{3}\right]=1
$$

Etant donnée une métrique riemannienne $g$ sur $M=\Gamma_{k} \backslash G$, la norme masse ||$_{\infty}$ sur $V_{1}=H_{1}(M, \mathbb{R})$ détermine une mesure mass* sur la variété

$$
J_{1} M=H_{1}(M, \mathbb{R}) / H_{1}(M, \mathbb{Z}),
$$

une métrique de Carnot-Carathéodory $d_{\infty}$ sur $G$, adaptée à la graduation $V_{1} \oplus \mathscr{G}^{2}$. Il reste à fixer une normalisation de la mesure de Haar sur $G$ : notons $\phi$ la 2 -forme sur $V_{1}$ dont l'intégrale est mass*; notons $\pi$ la projection $G \rightarrow G / G^{2}=V_{1}$; il y a une seule 1 -forme invariante $\omega$ sur $G$, nulle sur $V_{1}$, telle que

$$
d \omega=-\pi^{*} \phi
$$


par définition, la mesure vol est obtenue en intégrant la 3-forme invariante

$$
\pi^{*} \phi \wedge \omega
$$

(54) Calculons la mesure d'un domaine fondamental $P$ pour $\Gamma$ dans $G$. Soit $P^{\prime}$ le parallélogramme formé sur $v_{1}=\pi\left(\gamma_{1}\right)$ et $v_{2}=\pi\left(\gamma_{2}\right)$. Choisissons une section $s$ de au-dessus de $\dot{P}^{\prime}$ telle que

$$
\left.s^{*} \omega\right|_{\left(\partial P^{\prime}-0\right)}=0
$$

( $s$ a nécessairement une singularité en 0 ). Posons

$$
P=\left\{\left(\gamma_{3}\right)^{t} s(v): v \in P^{\prime}, \quad 0<t<1\right\}
$$

Alors

$$
\begin{aligned}
\operatorname{vol}(P) & =\int_{P} \pi^{*} \phi \wedge \omega=\int_{P^{\prime}}\left(\int_{P \cap \pi^{-1} v} \omega\right) \phi \\
& =\int_{P^{\prime}}\left(\int_{s(v)}^{\gamma_{3} s(v)} \omega\right) \phi \\
& =\operatorname{mass}^{*} J_{1} M \int_{1}^{\gamma_{3}} \omega
\end{aligned}
$$

Or la section $s$ permet de calculer l'intégrale de $\omega$ sur une fibre: le bord de $s$ est constitué de l'arc horizontal $s\left(\partial P^{\prime}-0\right)$, et d'un arc dans une fibre de l'origine 1 à $e \in G^{2}$, donc

$$
\int_{1}^{e} \omega=-\int_{s\left(P^{\prime}\right)} d \omega=\int_{P^{\prime}} \phi=\operatorname{mass}^{*} J_{1} M
$$

Il faut donc comparer $e$ à $\gamma_{3}$. Notons $\sigma$ l'automorphisme de $G$ qui vaut -id sur $V_{1}$ et id sur $\mathscr{G}^{2}$. Il préserve la forme $\omega$, donc les courbes horizontales. En particulier les relèvement horizontaux des droites de $V_{1}$, donc on peut écrire

$$
\sigma s\left(t v_{1}\right)=s\left(t v_{1}\right)^{-1}
$$

Ainsi, si on pose

$$
s\left(v_{1}\right)=a, \quad s\left(v_{1}+v_{2}\right)=a b, \quad s\left(v_{2}\right)=a b c, \quad e=a b c d,
$$

on a $\sigma a=a^{-1}, \sigma b=b^{-1}$ etc. D'autre part, les courbes horizontales

$$
t \mapsto s\left(v_{1}+v_{2}-t v_{1}\right) \quad \text { et } \quad t \mapsto a b \sigma s\left(t v_{1}\right)
$$

ont même origine et même image par $\pi$, donc sont égales, ce qui donne

$$
a b s\left(v_{2}\right)=a b c=a b \sigma(a)
$$

d'où

$$
c=a^{-1}, \quad d=b^{-1}, \quad e=[a, b] .
$$

Enfin, comme $a=\gamma_{1}$ et $b=\gamma_{2}$ modulo $G^{2}$, nous obtenons

$$
e=\left[\gamma_{1}, \gamma_{2}\right]=\gamma_{3}^{k}
$$

En comparant $\left({ }^{*}\right),\left({ }^{* *}\right),\left({ }^{* *}\right)$, nous concluons que

$$
\operatorname{vol}(P)=\frac{1}{k}\left(\operatorname{mass}^{*} J_{1} M\right)^{2} \text {. }
$$


(55) La mesure mass* est définie de façon à avoir la propriété suivante: pour tout parallélogramme $P$, le produit des distances entre les faces opposées est au moins égal à mass* $(P)$.

De manière équivalente, définissons mass* comme l'intégrale d'une deux forme $\phi$ de norme 1 , où la norme d'une deux-forme $\psi$ est définie par

$$
|\psi|=\inf \{|\alpha \| \beta|: \psi=\alpha \wedge \beta\} .
$$

et | | désigne la norme duale induite par ||$_{\infty}$ sur les formes linéaires.

(56) LeMme. Soit $\phi$ une 2-forme de norme 1 pour ||$_{\infty}$. Ecrivons $\phi=\alpha \wedge \beta$ où $|\alpha|=|\beta|=1$. Notons $l^{1}$ et $l^{\infty}$ les normes

$$
\begin{aligned}
& l^{\infty}(x)=\sup \{|\alpha(x)| ;|\beta(x)|\} \\
& l^{1}(x)=|\alpha(x)|+|\beta(x)|
\end{aligned}
$$

Alors

$$
l^{\infty} \leq||_{\infty} \leq l^{1},
$$

et les 2-formes correspondantes, de norme 1 et de même orientation que $\phi$, sont données par

$$
\phi^{1}=2 \phi, \quad \phi^{\infty}=\phi .
$$

Démonstration. Remarquer qu'il existe toujours une décomposition $\phi=\alpha \wedge \beta$ réalisant la borne inférieure $|\phi|=|\alpha||\beta|$. Soit $(a, b)$ la base de $V_{1}$, duale de $(\alpha, \beta)$; alors $|a|_{\infty}=|b|_{\infty}=1$; en effet,

$$
1=\alpha(a) \leq|\alpha \| a|_{\infty} \leq|a|_{\infty} ;
$$

si on avait $|a|_{\infty}>1$, il existerait un hyperplan séparant $a$ de la boule unité, c'est-à-dire, une forme linéaire $\alpha^{\prime}$ telle que $\alpha^{\prime}(a)=1$, mais $\left|\alpha^{\prime}\right|<1$; alors

$$
\alpha^{\prime} \wedge \beta=\alpha \wedge \beta=\phi,
$$

ce qui contredit la propriété de minimum de $(\alpha, \beta)$. Par conséquent, la boule unité de ||$_{\infty}$ contient $a, b,-a,-b$ donc leur enveloppe convexe, qui est la boule unité de $l^{1}$.

Enfin, pour tout $x$,

$$
l^{\infty}(x)=\sup \{|\alpha(x)|,|\beta(x)|\} \leq|x|_{\infty} .
$$

Un calcul élémentaire montre que $|\alpha \wedge \beta|=1$ pour $l^{\infty}$; on remarque, pour $l^{1}$, que cette norme admet une isométrie sur $l^{\infty}$ de déterminant 2 .

(57) Proposition. Notons $\nu^{*}$ le volume de la boule unité pour la métrique de Carnot-Carathéodory $d_{\infty}$ sur $G$, induite par ||$_{\infty}$, notons $\nu^{* 1}$ le volume correspondant pour la norme l $^{1}$. Alors

$$
\frac{1}{4} \nu^{* 1} \leq \nu^{*} \leq \nu^{* 1} .
$$

Démonstration. Notons $I=I(v)$ l'intervalle de la fibre $\pi^{-1}(v)$ se trouvant dans la boule unité $B_{\infty}(1)$; par définition,

$$
\nu^{*}=\operatorname{vol} B_{\infty}(1)=\int_{V_{1}} z(v) \phi
$$

où $z(v)=\int_{I} \omega$. Remarquons que, si $q=q(v)$ est l'unique point de la fibre en $v$ tel que $\sigma q=q^{-1}$, alors $I(v)$ est invariant par l'isométrie $x \mapsto q \sigma(x)$. 
Comparons $z$ aux quantités analogues $z^{\infty}$ et $z^{1}$ relatives à $l^{\infty}$ et $l^{1}$. Si $I(v)=$ $(p, q \sigma p)$ est un intervalle d'extrémités $p$ et $q \sigma p$, et si $c$ est une courbe de longueur 1 reliant l'origine 1 à $p$, alors

$$
\operatorname{long}_{\infty}(c) \leq \operatorname{long}(c)=1 \text {, }
$$

donc l'intervalle $I^{\infty}(v)$ contient $(p, q \sigma p)$, d'où

$$
z^{\infty}(v) \geq \int_{p}^{q \sigma p} \omega^{\infty}=\int_{I(v)} \omega=z(v) .
$$

Inversement, si $I^{1}(v)=\left(p^{1}, q \sigma p^{1}\right)$, et si $c^{1}$ est une courbe de longueur (relative à $l^{1}$ ) égale à 1 , reliant 1 à $p^{1}$, alors $I(v)$ contient $\left(p^{1}, q \sigma p^{1}\right)$, d'où

$$
z(v) \geq \int_{p^{1}}^{q \sigma p^{1}} \omega=\frac{1}{2} \int_{I^{1}(v)} \omega^{1}=\frac{1}{2} z^{1}(v) .
$$

Par conséquent,

$$
\begin{aligned}
& \nu^{* \infty}=\int_{l^{\infty}<1} z^{\infty}(v) \geq \int_{l^{\infty}<1} z(v) \phi \geq \nu^{*}, \\
& \nu^{* 1}=\int_{l^{1}<1} z^{1}(v) \phi^{1} \leq \int_{l^{1}<1} 2 z 2 \phi \leq 4 \nu^{*} .
\end{aligned}
$$

Enfin, $\nu^{* \infty}=\nu^{* 1}$, car les normes $l^{\infty}$ et $l^{1}$ sont isométriques.

(58) Rassemblant l'expression du volume asymptotique donnée en 51 , le volume du domaine fondamental donné en 54 , et l'inégalité 56 , nous concluons:

Proposition. Soit $(M, g)$ une variété riemannienne compacte, dont le groupe fondamental est isomorphe au sous-groupe $\Gamma_{k} d u$ groupe de Heisenberg. Le volume asymptotique s'écrit

$$
\operatorname{vol}_{\infty}(g)=k \nu^{*} \operatorname{vol}(g)\left(\text { mass } * J_{1} M\right)^{-2}
$$

où la constante $\nu^{*}$, qui ne dépend que de la variété de Jacobi, satisfait à

$$
0.22 \leq \nu^{*} \leq 0.89 \text {. }
$$

\section{Géodésiques fermées}

(59) Soit $g$ une métrique riemannienne sur la nilvariété $M=\Gamma \backslash G$, où $G$ est un groupe de Lie nilpotent. A chaque point $m$ de $M$ correspond une norme $|\gamma|=$ $d(\tilde{m}, \gamma \tilde{m})$ sur $\Gamma$. Alors le nombre $|\gamma|$ est la longueur minimum d'un lacet géodésique en $m$, représentant la classe d'homotopie $\gamma$; par conséquent, la fonction

$$
N(R)=\operatorname{card}\{\gamma \in \Gamma:|\gamma| \leq R\}
$$

est le nombre de lacets géodésiques en $m$, de longueur inférieure à $R$, dont l'existence est imposée par la structure du groupe fondamental.

(60) Proposition. Notons $\Gamma^{1}=\Gamma, \Gamma^{i+1}=\left[\Gamma, \Gamma^{i}\right]$ les sous-groupes de commutateurs successifs, notons

$$
p(i)=\sum_{j=i}^{\infty} j \operatorname{rang}\left(\Gamma^{i} / \Gamma^{j+1}\right)
$$


Alors la limite

$$
\lim _{R \rightarrow \infty} R^{-p(i)} \operatorname{card}\left\{\gamma \in \Gamma^{i}:|\gamma| \leq R\right\}
$$

existe toujours.

Démonstration. D'après le corollaire 48 , nous pouvons remplacer $|\gamma| \operatorname{par} d_{\infty}(1, h \gamma)$. D'après Malcev, le groupe discret $\Gamma^{i}$ est cocompact dans le groupe des commutateurs $G^{i} \subset G$; fixons un domaine fondamental $P$ pour $\Gamma^{i}$ dans $G^{i}:$ les $h(\gamma P)$ recouvrent $G_{\infty}^{i}$, et

$$
\operatorname{diam}_{\infty}(h \gamma P)=o\left(d_{\infty}(1, h \gamma)\right),
$$

donc dénombrer les $\gamma \in \Gamma^{i}$ tels que $d_{\infty}(1, \gamma) \leq R$ revient à calculer le volume des boules de $G_{\infty}^{i}$, pour la distance $d_{\infty}$ induite par $G_{\infty}$. Or $G_{\infty}^{i}$ est invariant sous les dilatations $\delta_{t}$ de $G_{\infty}$, et la mesure de Haar de $G_{\infty}^{i}$ est homogène de degré $p(i)$ pour le groupe $\delta_{t}$.

(61) Notons $C$ l'ensemble des classes d'homotopie libre de courbes fermées dans $M$, ou, ce qui revient au même, des classes de conjugaison dans $\Gamma$. Notons $|c|$ la longueur minimum d'une géodésique fermée représentant la classe $c$. Alors la fonction

$$
N_{c}(R)=\operatorname{card}\{c \in C:|c| \leq R\}
$$

donne le nombre topologique de géodésiques périodiques de longueur inférieure à $R$. Clairement, pour toute classe de conjugaison $c$ :

$$
|c| \leq \inf \{|\gamma|: \gamma \in c\} \leq|c|+2 \operatorname{diam}(g),
$$

par conséquent, si $\Gamma^{r}$ est le dernier groupe de commutateurs non nul, la limite

$$
\lim _{R \rightarrow+\infty} R^{-q} \text { card }\left\{c \in \Gamma^{r}:|c| \leq R\right\}
$$

existe, où $q=r$ rang $\left(\Gamma^{r}\right)$. La plupart de ces géodésiques périodiques sont primitives: en effet, les itérées d'une courbe $\gamma$ ne réalisent pas la longueur minimum dans leur classe d'homotopie:

(62) Proposition. Soit $\gamma \in \Gamma^{i}-\Gamma^{i+1}$; la longueur minimum d'un lacet homotope à l'itérée $\gamma^{k}$ croît comme $k^{1 / i}$, i.e. la limite

$$
\lim _{k \rightarrow+\infty}\left|\gamma^{k}\right| k^{-1 / i}
$$

existe.

Démonstration. En effet, considérons $\gamma \in G_{1}$, et écrivons $\gamma=\exp _{1}\left(z_{i}+\cdots+z_{r}\right)$. Posons $t=k^{1 / i} ;$ alors $\gamma^{k}=\exp _{1}\left(k z_{1}+\cdots+k z_{r}\right) ; \delta_{1 / t} \gamma^{k}=\exp _{t}\left(z_{i}+\cdots+k t^{-r} z_{r}\right)$ tend vers $\exp _{\infty} z_{i} \in G_{\infty}$, donc d'après la proposition $41,(1 / t)\left|\gamma^{k}\right|$ tend vers $d_{\infty}\left(1, \exp _{\infty} z_{i}\right) \neq 0$ lorsque $k$ tend vers l'infini.

(63). Corollaire. Soit $(M, g)$ une variété riemannienne telle que $\Gamma=\pi_{1}(M)$ soit cocompact dans le groupe d'Heisenberg de dimension 3. Notons $N_{0}(R)$ le nombre topologique de géodésiques périodiques de longueur inférieure à $R$, primitives et 
homologues à 0 . Alors, pour toute métrique riemannienne, la limite

$$
\lim _{R \rightarrow+\infty} N_{0}(R) / R^{2}
$$

existe.

Démonstration. Notons $\zeta$ un générateur de $\Gamma^{2}$; pour chaque $k \in \mathbb{Z}$, choisissons une géodésique fermée de longueur minimum dans la classe d'homotopie libre $\zeta^{k}$, notée $c(k)$, et montrons que l'équation

$$
c(k)=l \text {-ième itérée d'une courbe } c^{\prime}, \quad l \neq 1 .
$$

n'a qu'un nombre fini de solutions. Remarquons que $c(k) \doteq c^{\prime l}$ entraîne que $k=l m$, et que $c^{\prime}$ est de longueur minimum dans sa classe d'homotopie libre, d'où, si $d=$ diamètre $(g)$,

$$
\left|\zeta^{k}\right| \geq l\left(\left|\zeta^{m}\right|-2 d\right)
$$

Fixons un $\varepsilon>0$ tel que $(1+\varepsilon) /(1-\varepsilon)<\sqrt{2}$; d'après le $\S 62$, il existe un $A$ tel que, si $\left|\zeta^{k}\right|>A$.

$$
(1-\varepsilon) \Delta \sqrt{k} \leq\left|\zeta^{k}\right| \leq(1+\varepsilon) \Delta \sqrt{k}, \quad \text { où } \Delta=d_{\infty}(1, \zeta) .
$$

Fixons un $B>A$ tel que $(1+\varepsilon) /(1-\varepsilon)(B / B-2 d)<\sqrt{2}$. Alors, pour chacun des $m$ tels que $\left|\zeta^{m}\right| \leq B$, l'équation $c(l m)=c(m)^{l}$ n'a qu'un nombre fini de solutions, d'après $\S 62$ appliqué à $\zeta^{m}$.

$\mathrm{Si}$, au contraire, $\left|\zeta^{m}\right|>B$, et si $\left|\zeta^{k}\right|>A$, alors

$$
\left|\zeta^{k}\right| \leq((1+\varepsilon) /(1-\varepsilon))\left|\zeta^{m}\right| \sqrt{l}
$$

d'où

$$
l<\left[\left((1+\varepsilon) /(1-\varepsilon)\left|\zeta^{m}\right|\right) /\left(\left|\zeta^{m}\right|-2 d\right)\right]^{2}<2
$$

donc il n'y a pas de solution dans ce cas.

(64) Jusqu'à présent, nous savons dénombrer seulement les géodésiques fermées du dernier sous-groupe de commutateurs $\Gamma^{r}$. Pour dénombrer les géodésiques dans $\Gamma^{r-1}-\Gamma^{r}$, il faut connaître les classes de conjugaison dans $\Gamma$; c'est pourquoi nous ne donnons de résultat qu'en dimension 3 .

Proposition. Supposons $\Gamma=\pi_{1}(M)$ cocompact dans le groupe d'Heisenberg; la limite

$$
\lim _{R \rightarrow+\infty} R^{-2} \text { card }\{c \in C, c \text { non homologue à } 0:|c| \leq R\}
$$

existe toujours.

Nous allons utiliser le fait élémentaire suivant:

(65) Scholie. Soit a $(R)$ une suite de nombres positifs telle que

(i) pour tout $R$ et tout entier $p, a(p R) \geq p^{2} a(R)$;

(ii) pour tout $r$ fixé, $a(R)-a(R-r)=o\left(R^{2}\right)$ lorsque $R$ tend vers $+\infty$.

Alors la limite $\lim _{R \rightarrow+\infty} R^{-2} a(R)$ existe.

Posons $s=\lim \sup a(R) / R^{2}$, et soit $q$ assez grand pour que

$$
a(q) / q^{2} \geq s-\varepsilon
$$


Par (ii), il existe un $A$ tel que, pour $p>A$ et pour tout $r, 0 \leq r<q$,

$$
a(p q)-a(p q-r)<\varepsilon(p q)^{2}
$$

d'où

$$
a(p q-r) /(p q-r)^{2} \geq a(p q) /(p q)^{2}-\varepsilon \geq a(q) / q^{2}-\varepsilon \geq s-2 \varepsilon
$$

Démonstration de la proposition 64. Pour chaque classe de conjugaison $c$, fixons un représentant $\gamma(c)$ tel que $d_{\infty}(1, \gamma(c))$ soit minimum. D'après le corollaire 48 et l'inégalité du $\$ 61$, nous pouvons remplacer, dans l'énoncé, le nombre $|c|$ par $d_{\infty}(1, \gamma(c))$.

L'image $\pi(c)$ dans $V_{1}$ est bien définie; $c$ non homologue à 0 se traduit par $\pi(c) \neq 0$. Fixons une base $(a, b)$ du réseau $\pi(\Gamma)$ : alors, au-dessus du point $m a+n b$ de $\pi(\Gamma)$, il y a exactement $m \wedge n=$ pgcd de $m$ et $n$ classes de conjugaison. Par conséquent, si $\pi(c)=m a+n b$,

$$
d_{\infty}(1, \gamma(c)) \leq|m a+n b|_{\infty}+\Delta \sqrt{m \wedge n+1}
$$

où $\Delta=d_{\infty}(1, \zeta)$ pour un générateur $\zeta$ de $\Gamma^{2}$.

Notons $C(R)$ l'ensemble des classes de conjugaison $c$ telles que

$$
d\left(1, \gamma(c) \zeta^{t}\right) \leq R \quad \text { pour tout } t \in\left[-\frac{1}{2}, \frac{1}{2}\right]
$$

et posons

$$
a(R)=\operatorname{card} C(R) .
$$

Vérification de (i). Soit $j$ un entier relatif, $|j| \leq p^{2}-\frac{1}{2}, c \in C(R)$; alors, pour tout $t \in\left[-\frac{1}{2}, \frac{1}{2}\right]$, on a

$$
d_{\infty}\left(1, \delta_{p} \gamma(c) \zeta^{j+t}\right)=p d_{\infty}\left(1, \gamma(c) \zeta^{j+t / p^{2}}\right) \leq p R
$$

donc la classe de conjugaison de $\delta_{p}(c) \zeta^{j}$ est dans $C(p R)$. Or, lorsque $c$ varie dans $C(R)$ et $j$ de $-\left(p^{2}-1\right) / 2$ à $\left(p^{2}-1\right) / 2$, les classes de conjugaison $\delta_{p} c \zeta^{j}$ obtenues sont toutes distinctes, donc

$$
\begin{aligned}
a(p R) & \geq\left(p^{2}-1\right) a(R), & & \text { si } p \text { est pair, } \\
& \geq p^{2} a(R) & & \text { si } p \text { est impair. }
\end{aligned}
$$

Vérification de (ii). Comme $d_{\infty}(1, \gamma(c)) \geq|\pi(c)|_{\infty}$, tous les $\pi(c)$, pour $c \in C(R)$, sont dans

d'où l'inégalité

$$
B(R)=\left\{a m+b n:|a m+b n|_{\infty} \leq R\right\},
$$

$$
a(R) \leq \sum_{B(R)} m \wedge n
$$

inversement, si $|a m+b n|_{\infty}+\Delta \sqrt{m \wedge n+2} \leq R-r$, pour toute classe $c$ telle que $\pi(c)=m a+n b$ et tout $t \in\left[-\frac{1}{2}, \frac{1}{2}\right]$, on a

$$
d_{\infty}\left(1, \gamma(c) \zeta^{t}\right) \leq R-r
$$

soit $c \in C(R)$; il vient donc

$$
a(R-r) \geq \sum\left\{m \wedge n:|m a+n b|_{\infty}+\Delta \sqrt{m \wedge n+2} \leq R-r\right\} \geq \sum_{B(S)} m \wedge n,
$$

pour un $S$ tel que $R-$ const. $\sqrt{R} \leq S \leq R$, car $m \wedge n \leq$ const. $|m a+n b|_{\infty}$. 
Nous pouvons donc majorer $a(R)-a(R-r)$ par $\sum_{p=1}^{R} p b_{p}$, où $b_{p}$ est le nombre de couples d'entiers $(\mu, \nu)$ premiers entre eux, et tels que

$$
S \leq p|\mu a+\nu b|_{\infty} \leq^{\cdot} R .
$$

Fixons une fonction $\varepsilon(R)$ tendant vers 0 . Pour $p \leq \varepsilon R$, nous majorons

$$
b_{p} \leq \operatorname{card}\left\{(\mu, \nu): S \leq p|\mu a+\nu b|_{\infty} \leq R\right\}
$$

au moyen du volume des boules dans $V_{1}$ : si celui-ci est en $\tau R^{2}$, et si $d$ est le diamètre d'un domaine fondamental de $\pi(\Gamma)$ dans $V_{1}$, alors

$$
p b_{p} \leq p \tau\left((R / p+d)^{2}-(S / p-d)^{2}\right)=\left(\left(R^{2}-S^{2}\right) / p+2 d(R+S)\right) .
$$

d'où

$$
\sum_{p=1}^{\varepsilon R} p b_{p} \leq \text { const. }\left(R \sqrt{R} \log (\varepsilon R)+\varepsilon R^{2}\right) .
$$

Pour $p>\varepsilon R$, nous majorons $p$ par $R$ et $\sum_{p=\varepsilon R}^{R} b_{p}$ par

$$
\begin{aligned}
& \quad \operatorname{card}\left\{(\mu, \nu): \text { il existe } p>\varepsilon R \text { tel que } p|\mu a+\nu b|_{\infty} \leq R\right\} \\
& \leq \operatorname{card}\left\{(\mu, \nu):|\mu a+\nu b|_{\infty} \leq \varepsilon^{-1}\right\} \leq \text { const. } \varepsilon^{-2}
\end{aligned}
$$

d'où

$$
\sum_{p=\varepsilon R}^{R} p b_{p} \leq \text { const. } R \varepsilon^{-2}
$$

Enfin, il vient

$$
a(R)-a(R-r) / R^{2} \leq \text { const. }\left(\varepsilon+1 / R \varepsilon^{2}+\log (\varepsilon R) / R\right),
$$

qui tend vers 0 lorsque $\varepsilon(R)=R^{-\frac{1}{3}}$, par exemple.

(66) En combinant les deux résultats précédents, nous concluons:

Proposition. Soit $\Gamma$ un sous-groupe discret cocompact du groupe de Lie nilpotent, non abélien, de dimension 3. Pour toute variété riemannienne $(M, g)$ dont le groupe fondamental est isomorphe à $\Gamma$, la limite

$$
\lim _{R \rightarrow+\infty} N_{c}(R) / R^{2}
$$

existe, où $N_{c}(R)$ désigne le nombre topologique (cf $\$ 33$ ) de géodésiques périodiques de longueur inférieure à $R$ dans $(M, g)$.

Appendice: torsion dans un groupe nilpotent de type fini (67). Le but de cet appendice est de démontrer la

Proposition. Dans un groupe nilpotent, de type fini, les éléments de torsion forment un sous-groupe fini.

(68) LEMME. Soit $\Gamma$ ungroupe; a et tdes éléments de $\Gamma, z=[a, t]$. Si a et $z$ commutent, alors, pour tout $n$, on a

$$
z^{n}=\left[a^{n}, t\right]
$$


Démonstration. En effet,

$$
\begin{aligned}
{\left[a^{n+1}, t\right] } & =a a^{n} t a^{-n} a^{-1} t^{-1}=a\left[a^{n}, t\right] t a^{-1} t^{-1} \\
& =\left[a^{n}, t\right][a, t]
\end{aligned}
$$

si $a$ et $\left[a^{n}, t\right]$ commutent.

(69) LEMME. Soit $\Gamma$ un groupe nilpotent, engendré par $\left\{a_{0}, \ldots, a_{h}\right\}$. Supposons que $\left\{a_{1}, \ldots, a_{h}\right\}$ engendrent un sous-groupe fini $F$; alors, si $a_{0}$ est de torsion, le groupe $\Gamma$ est fini.

Démonstration. Pour $z \in \Gamma$, posons

$$
S_{z}^{1}=\{z\}, \quad S_{z}^{i+1}=\left\{\left[a_{k}, t\right]: 0 \leqslant k \leqslant h, t \in S_{z}^{i}\right\}, \quad S z=\bigcup S_{z}^{i},
$$

notons $\Sigma z$ le sous-groupe engendré par $S z$. Pour tout $z$, l'ensemble $S z$ est fini. Il s'agit de montrer que $\Sigma a_{0}$ est fini: en effet, $\Sigma a_{0}$ est distingué dans $\Gamma$, et l'application

$$
F \rightarrow \Gamma / \Sigma a_{0}
$$

est surjective. Comme $\Gamma$ est nilpotent, il existe un $j$ tel que, si $i \geq j+1, S_{a_{0}}^{i}=\{1\}$. Alors, si $z \in S_{a_{0}}^{j}$, le groupe $\Sigma z$ est fini: il est engendré par $S z=\{z\}$; par hypothèse, $z=\left[a_{k}, t\right]$, et $\left[a_{k}, z\right] \in S_{a_{0}}^{j+1}$ est nul, donc

$$
z^{n}=\left[a_{k}^{n}, t\right]=1 \quad \text { pour } n \text { assez grand. }
$$

Montrons, par récurrence descendante sur $i$, que $\Sigma z$ est fini pour tout $z \in S_{a_{0}}^{i}$.

Fixons $z \in S_{a_{0}}^{i}$, notons $\Sigma^{m}$ le sous-groupe engendré par les $S\left[a_{k}, z\right]$, pour $k>0$, et les $S^{n}\left[a_{0}, z\right]$, pour $n \geq m$.

Alors $\Sigma^{m}$ est distingué dans $\Gamma$ : en effet, si $k>0$, et $t \in S\left[a_{k}, z\right]$,

$$
a_{l} t a_{l}^{-1}=\left[a_{l}, t\right] t \in \Sigma\left[a_{k}, z\right] \subset \Sigma^{m}
$$

pour $k=0, t \in S^{n}\left[a_{0}, z\right]$,

$$
a_{l} t a_{l}^{-1}=\left[a_{l}, t\right] t
$$

est dans le sous-groupe engendré par $S^{n}\left[a_{0}, z\right]$, donc dans $\Sigma^{m}$.

Le quotient $\Sigma^{m} / \Sigma^{m+1}$ est abélien: en effet, si $k>0$, et $t \in S\left[a_{k}, z\right]$,

$$
\left[a_{l}, t\right] \in S\left[a_{k}, z\right] \subset \Sigma^{m+1}
$$

pour $k=0$, et $t \in S^{n}\left[a_{0}, z\right]$,

$$
\left[a_{l}, t\right] \in S^{n+1}\left[a_{0}, z\right] \subset \Sigma^{m+1} .
$$

Le quotient $\Sigma^{m} / \Sigma^{m+1}$ est engendré par des éléments de torsion: en effet, il est engendré par

$$
S^{m}\left[a_{0}, z\right] \subset \Sigma\left[a_{0}, z\right]
$$

qui est fini, par l'hypothèse de récurrence, et le fait que $\left[a_{0}, z\right] \in S^{i+1} a_{0}$. Nous concluons que chaque quotient est fini, donc la finitude de $\Sigma^{1}$ résulte de celle du groupe engendré par les $S\left[a_{k}, z\right], k>0$. Par récurrence sur $k$, on voit donc que le groupe $\Sigma^{1}$ engendré par les $S\left[a_{k}, z\right], k \geq 0$, est fini.

Or $\Sigma^{1}$ est distingué, et le groupe quotient $\Sigma z / \Sigma^{1}$ est engendré par $z$. Ecrivons $z=\left[a_{k}, t\right]$, pour un $t \in S^{i-1} a_{0}$; comme $\left[a_{k}, z\right]=1$ modulo $\Sigma^{1}$, pour un entier $p$, $z^{p}=\left[a_{k}^{p}, t\right]=1 \bmod \Sigma^{1}$, donc le quotient $\Sigma z / \Sigma^{1}$ est fini, ce qui prouve que $\Sigma z$ est fini et achève la démonstration par récurrence. 
(70) Soit $\Gamma$ un groupe nilpotent, de type fini. Le lemme 69 montre que les éléments de torsion forment un sous-groupe, et que, s'il est de type fini, il est fini. La proposition 67 résulte donc du lemme suivant:

LEMME. Dans un groupe nilpotent, de type fini, tout sous-groupe est de type fini.

Démonstration. Raisonnons par récurrence sur l'ordre du groupe $\Gamma$. Notons $\Gamma^{2}$ le groupe des commutateurs, et $\pi: \Gamma \rightarrow \Gamma / \Gamma^{2}$. Par l'hypothèse de récurrence, pour tout sous-groupe $\Gamma^{\prime}, \Gamma^{\prime} \cap \Gamma^{2}$ et $\pi\left(\Gamma^{\prime}\right)$ sont de type fini, donc $\Gamma^{\prime}$ est de type fini.

\section{BIBLIOGRAPHIE}

[1] H. Bass. The degree of polynomial growth of finitely generated nilpotent groups. Proc. London Math. Soc. (3) 25 (1972), 603-614.

[2] V. Efremovič. The proximity geometry of Riemannian manifolds. Uspehi Mat. Nauk 8 (1953), 189.

[3] H. Federer \& W. H. Fleming. Normal and integral currents. Ann. of Math. 72 (1960), 458-520.

[4] B. Gaveau. Principe de moindre action, propagation de la chaleur et estimées sous-elliptiques sur certains groupes nilpotents. Acta Math. 139 (1977), 96-153.

[5] B. Gaveau. Systèmes hamiltoniens associés à certains opérateurs hypoelliptiques. Bull. Sci. Math. (1978), 203-229.

[6] R. Goodman. Nilpotent Lie Groups, structure and applications to Analysis. Lecture Notes 562. Springer-Verlag: Berlin, 1976.

[7] M. Gromov. Groups of polynomial growth and expanding maps. Publ. Math. de l'I.H.E.S. 53 (1981), 53-78.

[8] M. Gromov. Structures métriques pour les variétés riemanniennes, notes de cours rédigées par $\mathbf{J}$. Lafontaine et P. Pansu. CEDIC: Paris, 1981.

[9] M. Gromov. Filling riemannian manifolds. J. Diff. Geom. 18 (1983), 1-147.

[10] A. Kaplan. Riemannian nilmanifolds attached to Clifford Modules. Geometriae Dedicata 11 (1981), 127-136.

[11] A. Koranyi. Geometric properties of Heisenberg type groups. Preprint. (Washington Univ. at St Louis.)

[12] A. Malcev. On a class of homogeneous spaces. Isvestiya 13 (1949), 9-32. (Amer. Math. Soc. Transl. 39 (1951).)

[13] G. A. Margulis. Certain applications of ergodic theory to the investigation of manifolds of negative curvature. Funkc. Analiz i Prilozen. 3 (1969) N ${ }^{\circ} 4,89-90$.

[14] P. Pansu. Une inégalité isopérimétrique sur le groupe d'Heisenberg. C.R. Acad. Sci. Paris 295 (1982), 127-131.

[15] H. S. Süssmann. Orbits of families of vectorfields and integrability of distributions. Trans. A.M.S. 180 (1973), 171-188.

[16] A. Švarč. A volume invariant of coverings, Doklady Akad. Nauk SSSR 105 (1955), 32-34.

[17] J. Tits. Groupes à croissance polynomiale. Séminaire Bourbaki, exposé $\mathrm{N}^{\circ} 572$.

[18] J. Wolf. Growth of finitely generated solvable groups and curvature of riemannian manifolds. $J$. Diff. Geom. 2 (1968), 421-446.

[19] Encyclopedic Dictionary of Mathematics. Article $N^{\circ} 240$ : Lattice point problems. Math Soc. of Japan-MIT Press: Cambridge, U.S.A. 